Catalytic Hydroconversion of a High-Temperature Coal Tar over Two Attapulgite Powder-Supported Nickel Catalysts

Yang-Yang Zhang ${ }^{\text {a }}$, Xian-Yong Wei ${ }^{\text {a,b* }}$, Jing-Hui Lv ${ }^{\mathrm{c}}$, Zhi-Min Zong ${ }^{\text {a }}$

${ }^{a}$ Key Laboratory of Coal Processing and Efficient Utilization, Ministry of Education, China University of Mining \& Technology, Xuzhou 221116, Jiangsu, China

${ }^{b}$ State Key Laboratory of High-efficiency Utilization and Green Chemical Engineering, Ningxia University, Yinchuan 750021, Ningxia, China

${ }^{\mathrm{c}}$ Henan Key Laboratory of Coal Green Conversion, Henan Polytechnic University, Jiaozuo 454003, Henan, China

* Corresponding author. Tel: +86 51683885951.

E-mail address: wei_xianyong@163.com (X. Y. Wei). 


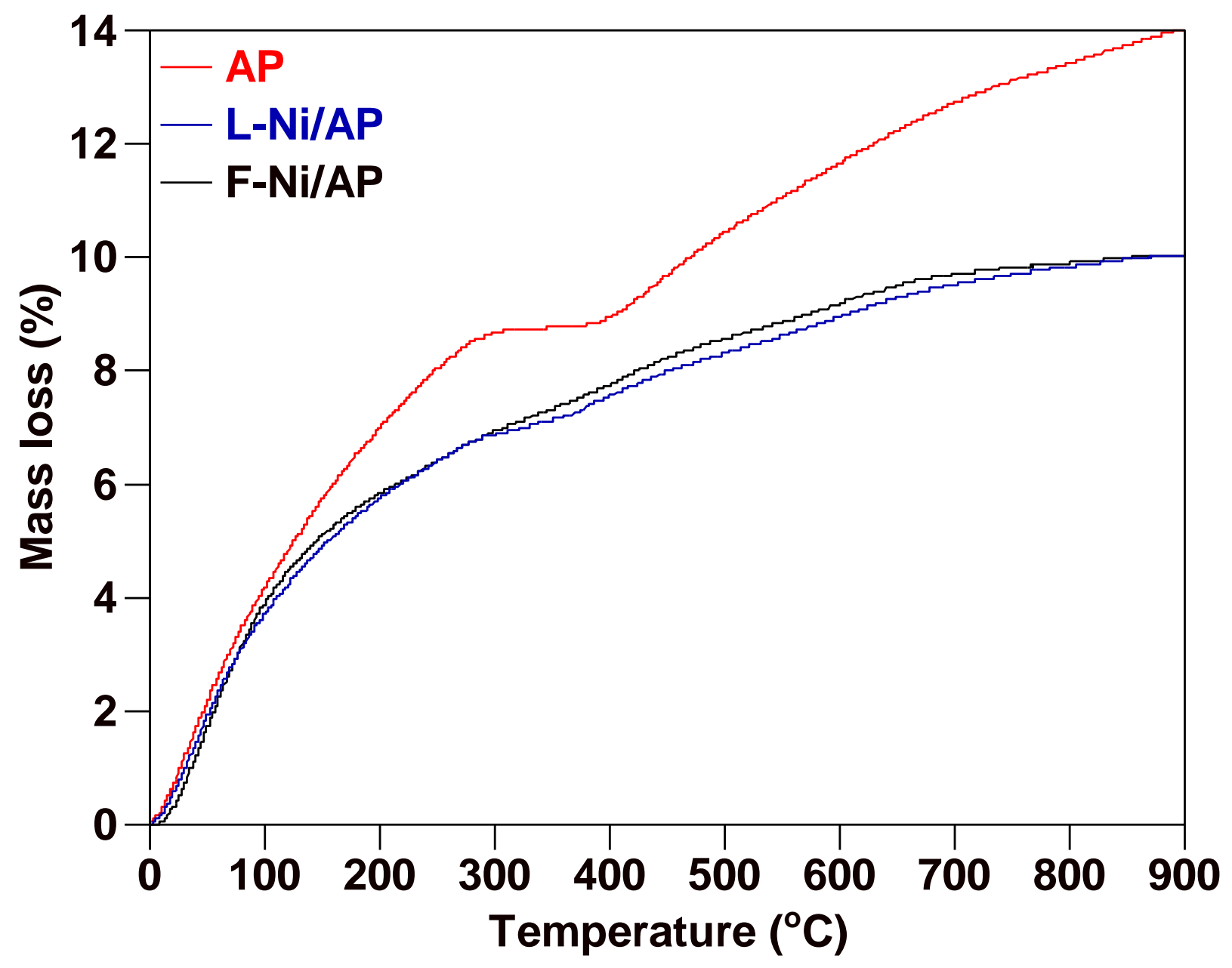

Figure S1. TG curves of AP, L-Ni/AP, and F-Ni/AP. 

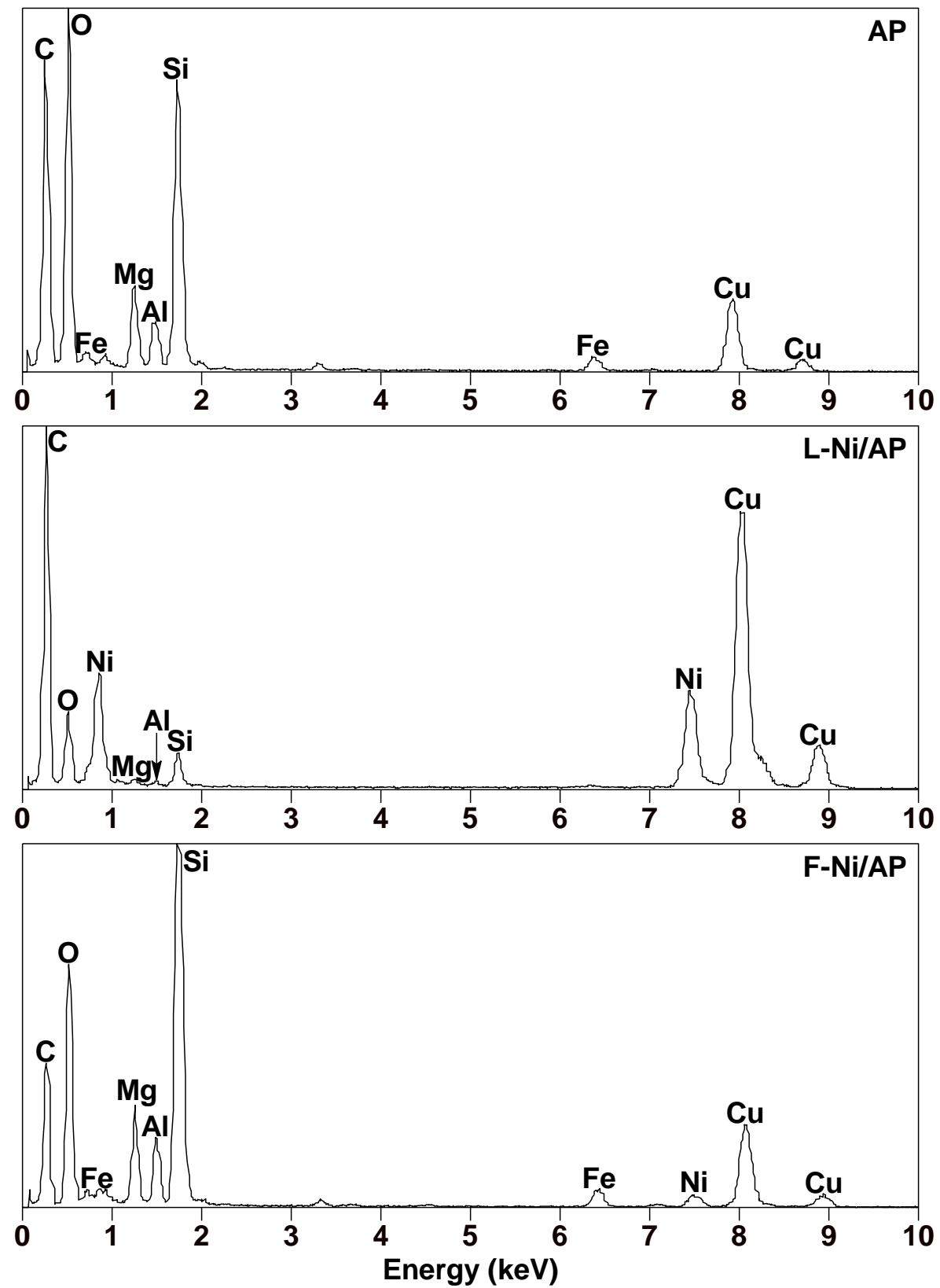

Figure S2. Energy dispersive spectrum of AP, L-Ni/AP, and F-Ni/AP. 

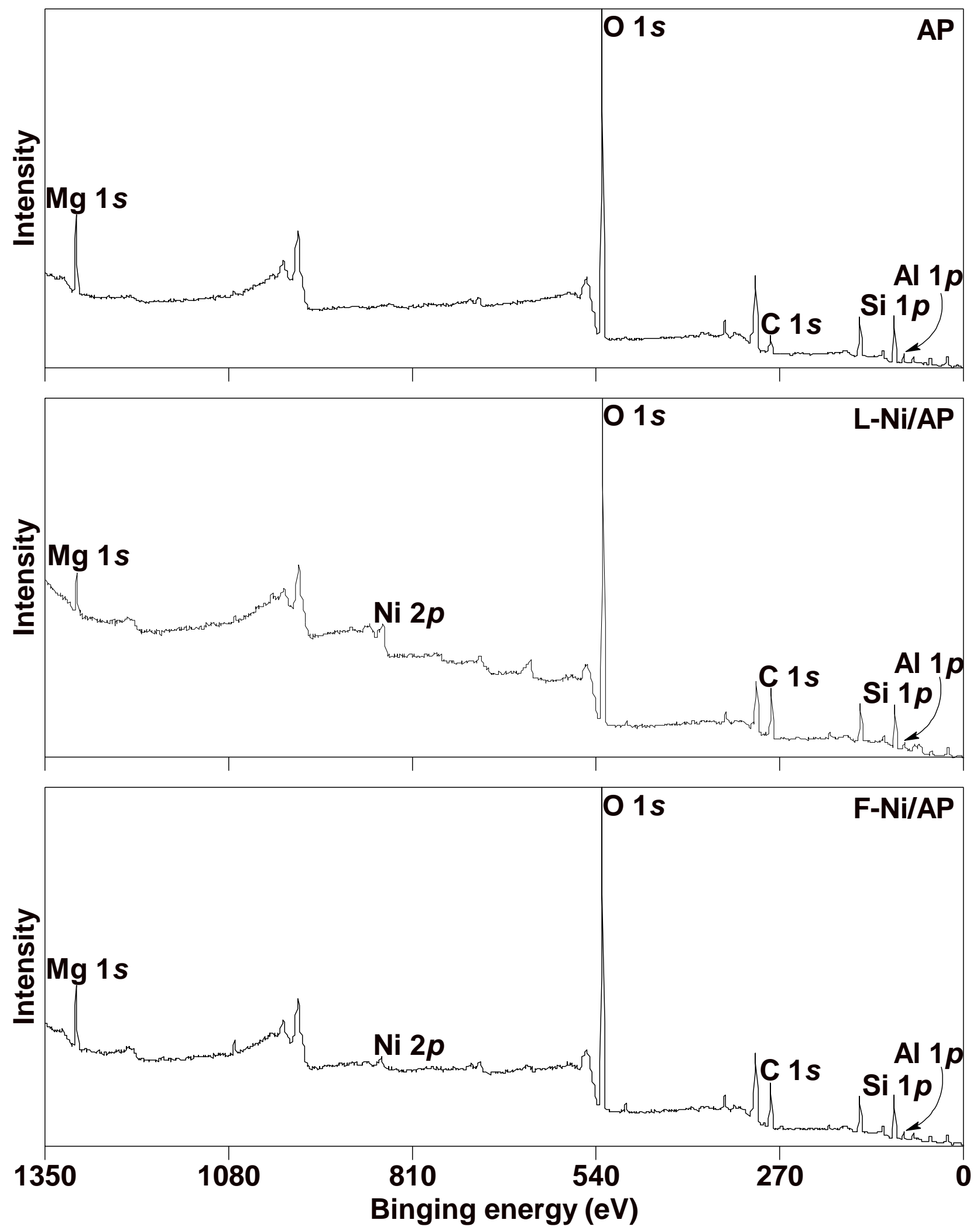

Figure S3. XRPES of AP, L-Ni/AP, and F-Ni/AP. 


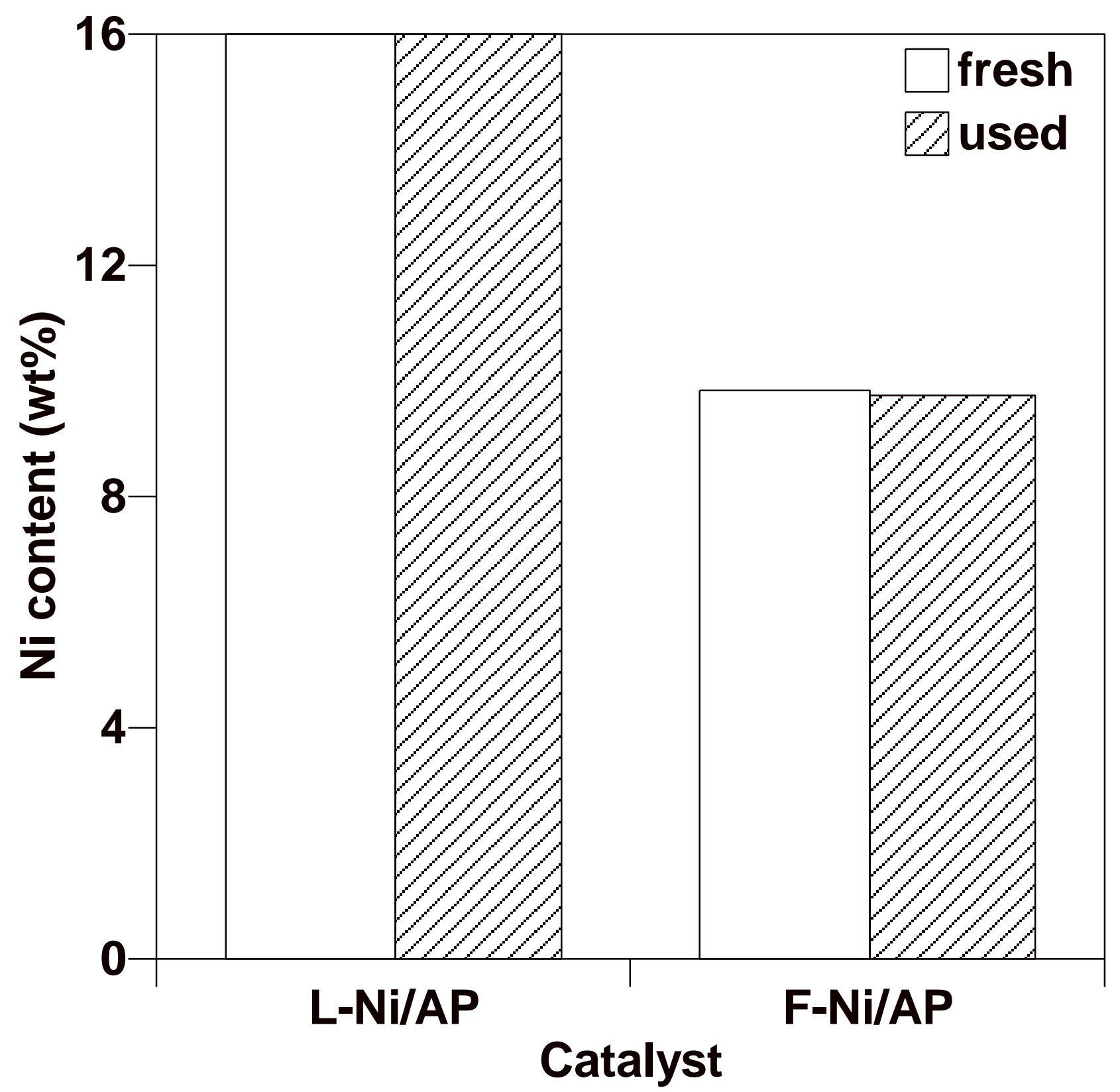

Figure S4. Ni content of fresh and used catalysts according to the analysis with ICP-MS. 


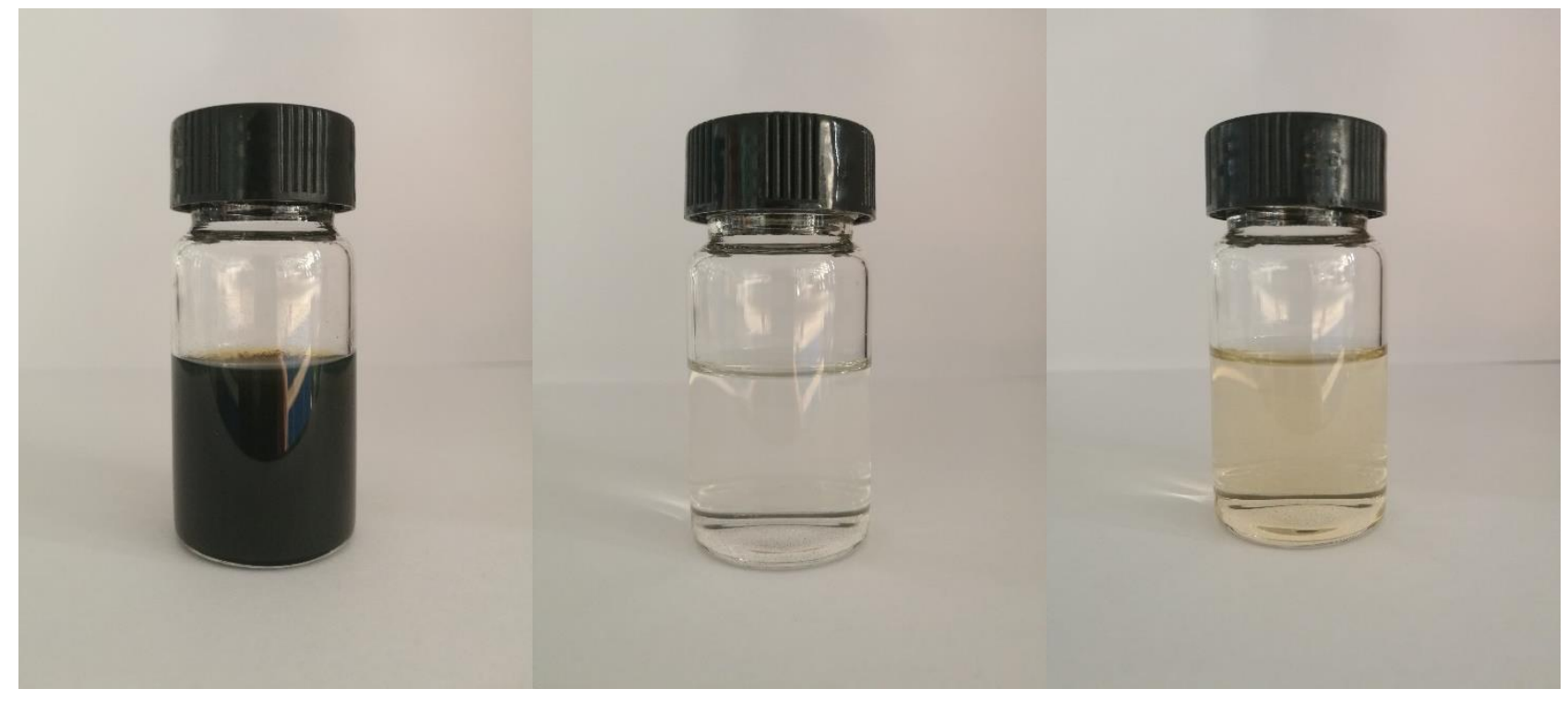

Figure S5. Photographs of the HTCT (left) and hydroconverted HTCT (middle and right). 


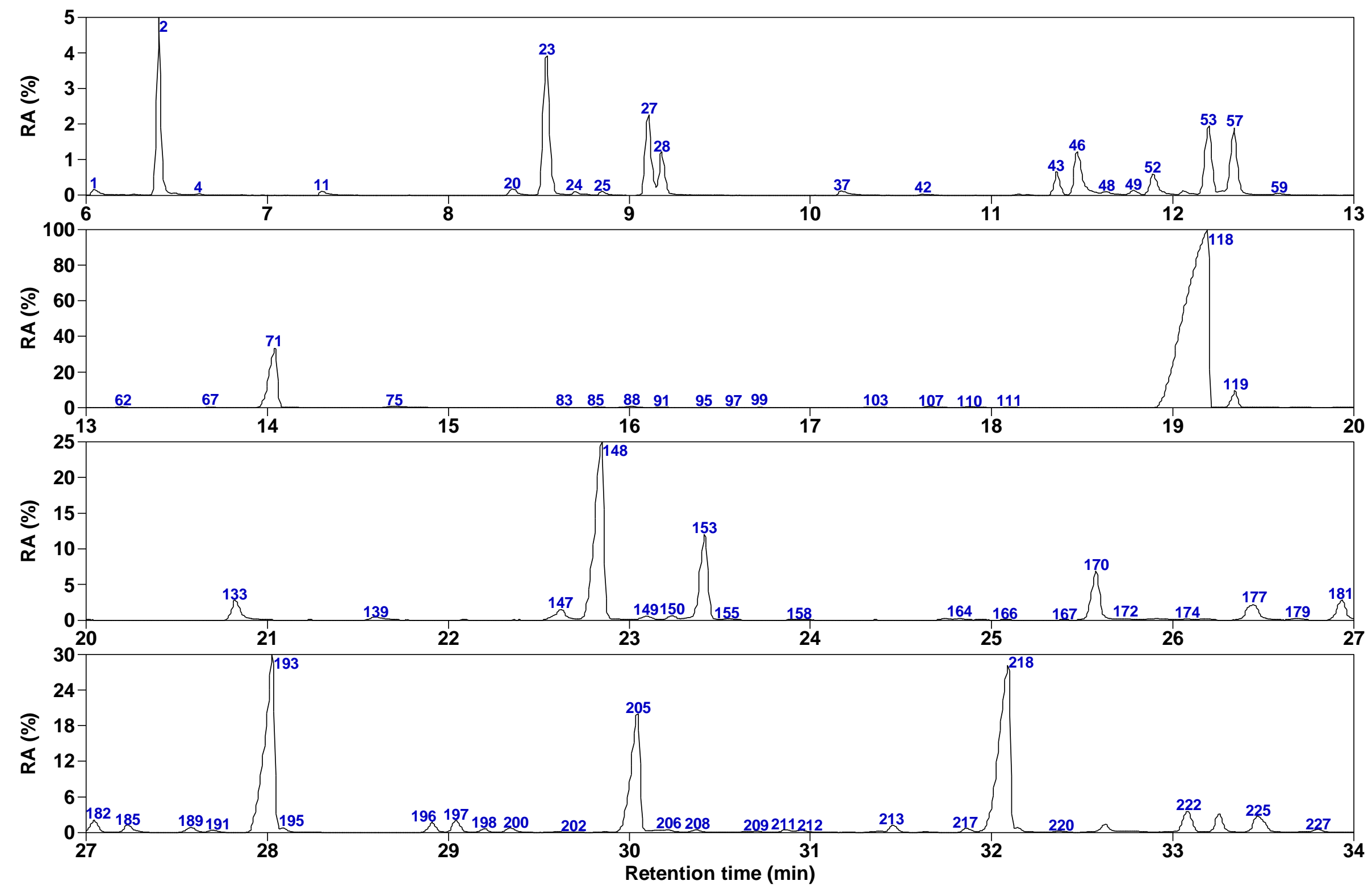

Figure S6. Total ion chromatogram of $\mathrm{SP}_{\mathrm{NCHC}}$. 

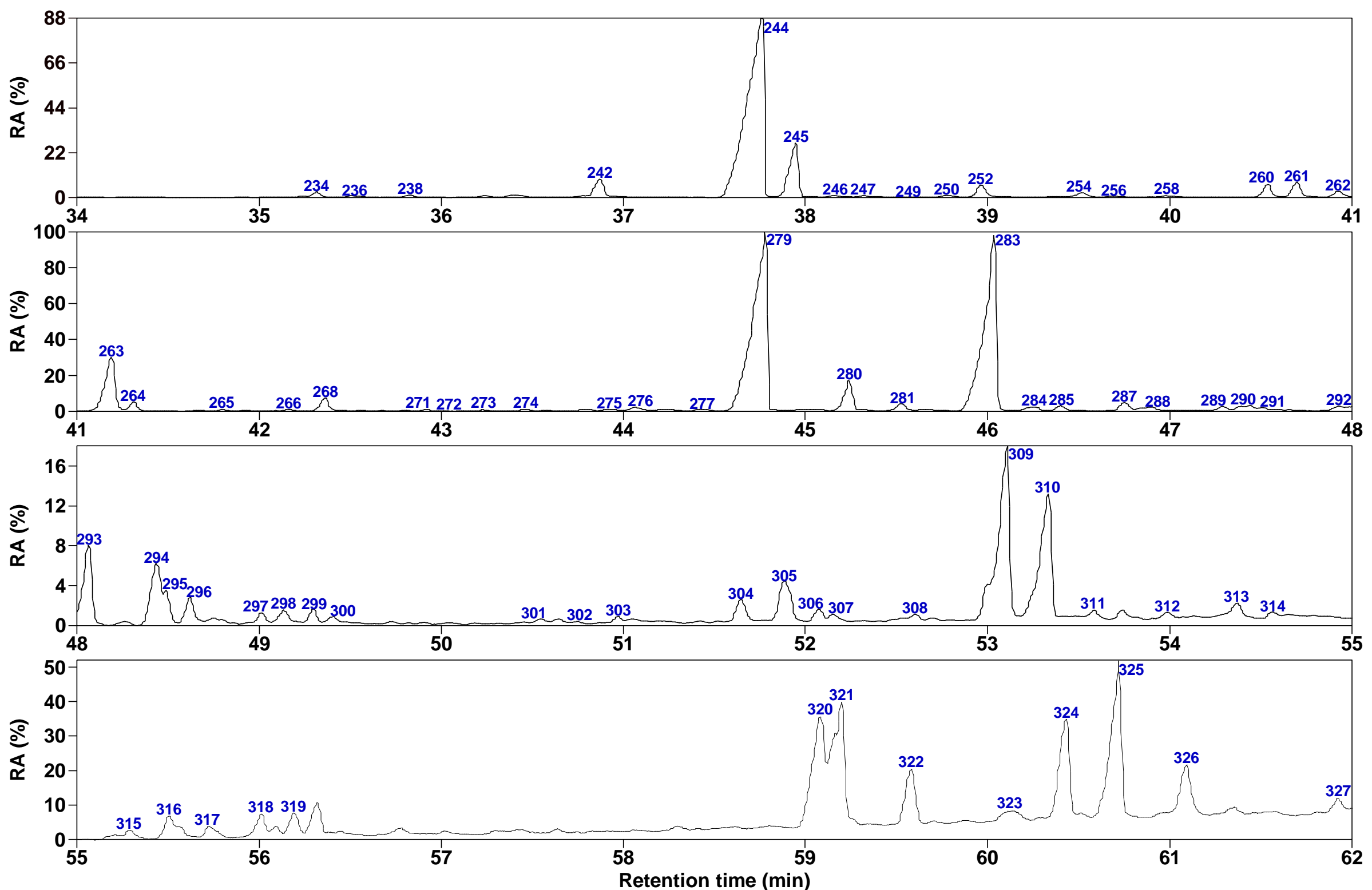

Figure S6. Total ion chromatogram of $\mathrm{SP}_{\mathrm{NCHC}}$ (continued). 

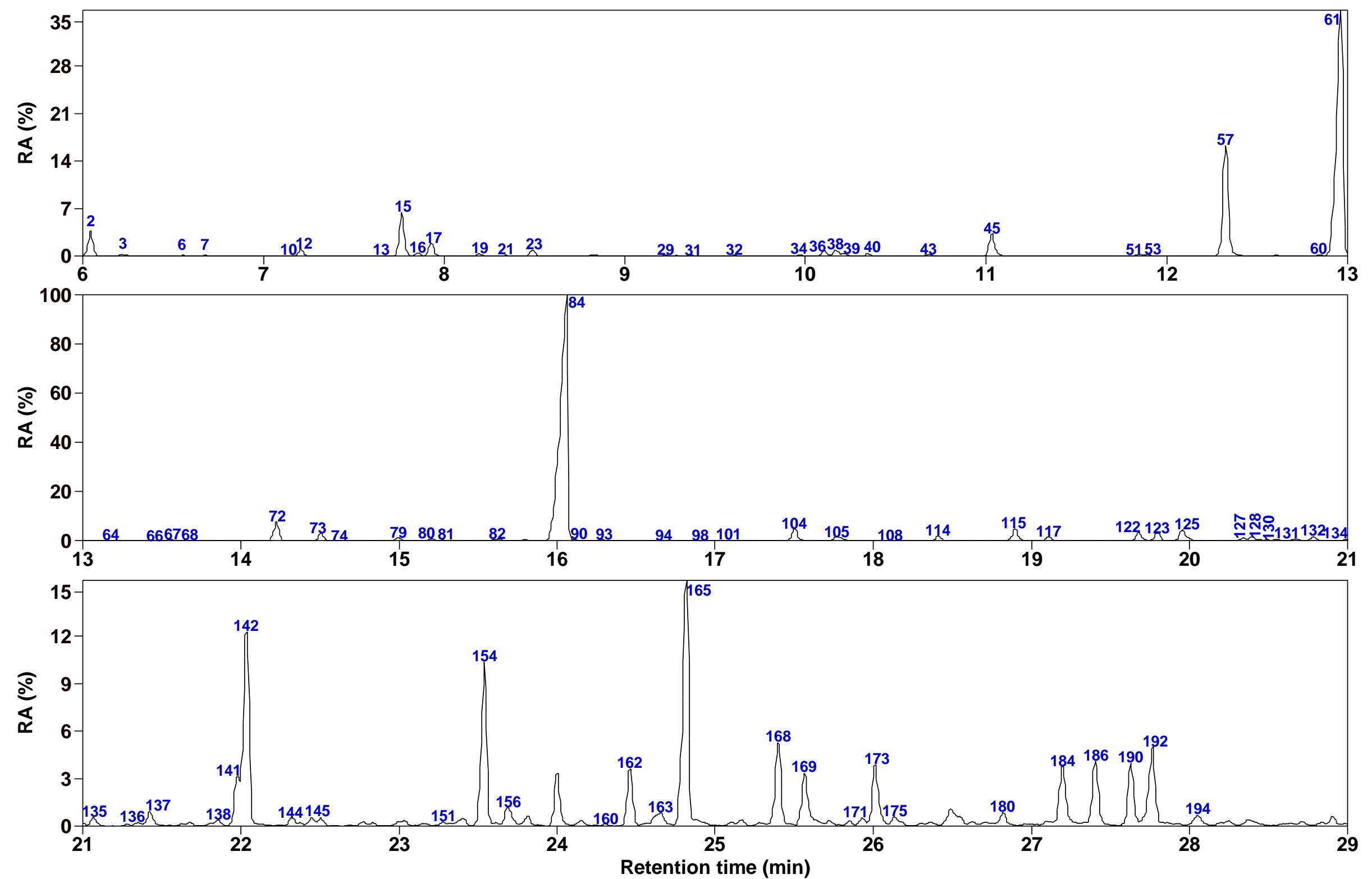

Figure S7. Total ion chromatogram of SP $\mathrm{CHC}_{\mathrm{C}-\mathrm{L}}$. 

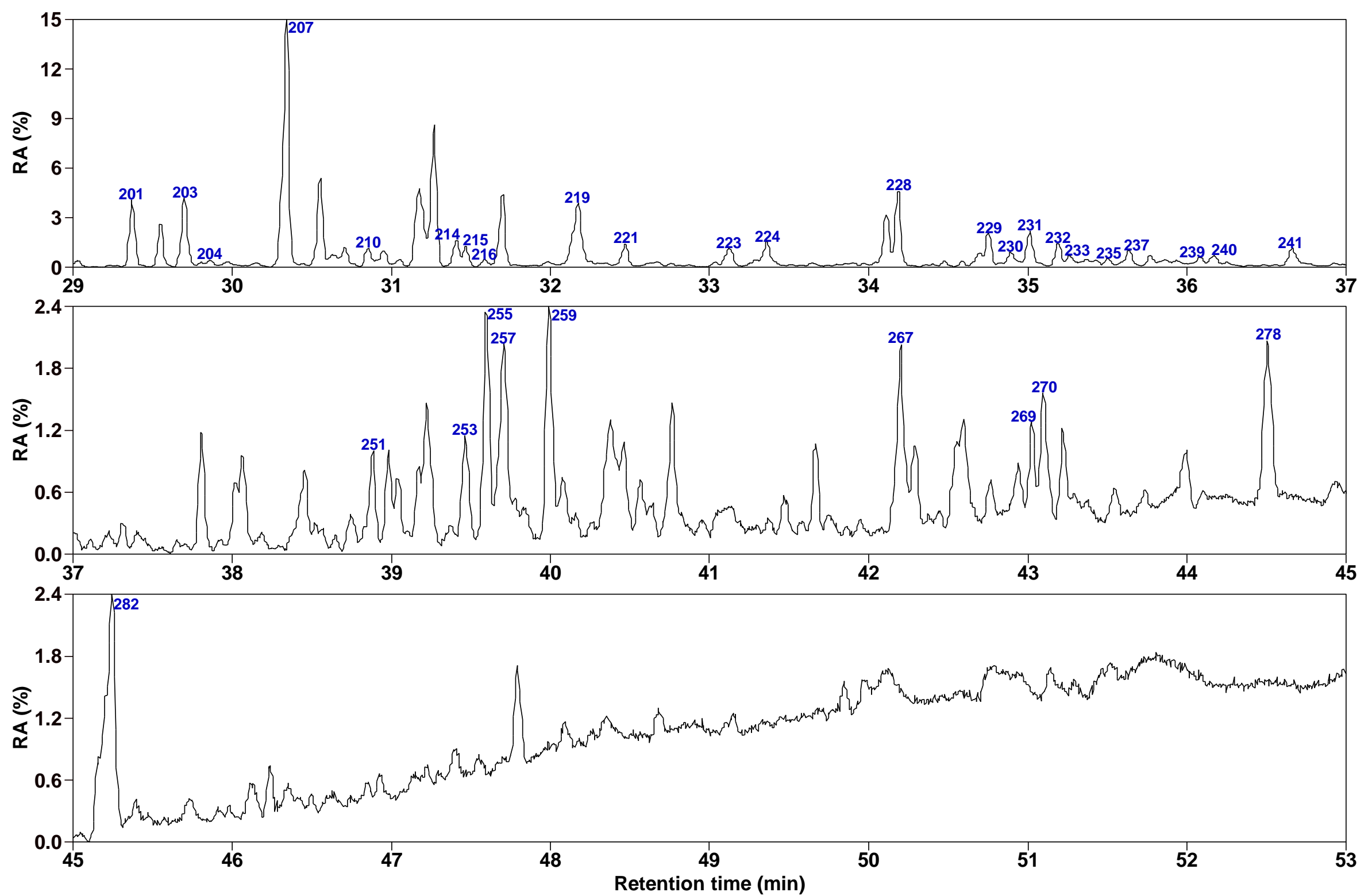

Figure S7. Total ion chromatogram of $\mathrm{SP}_{\mathrm{CHC}-\mathrm{L}}$ (continued). 

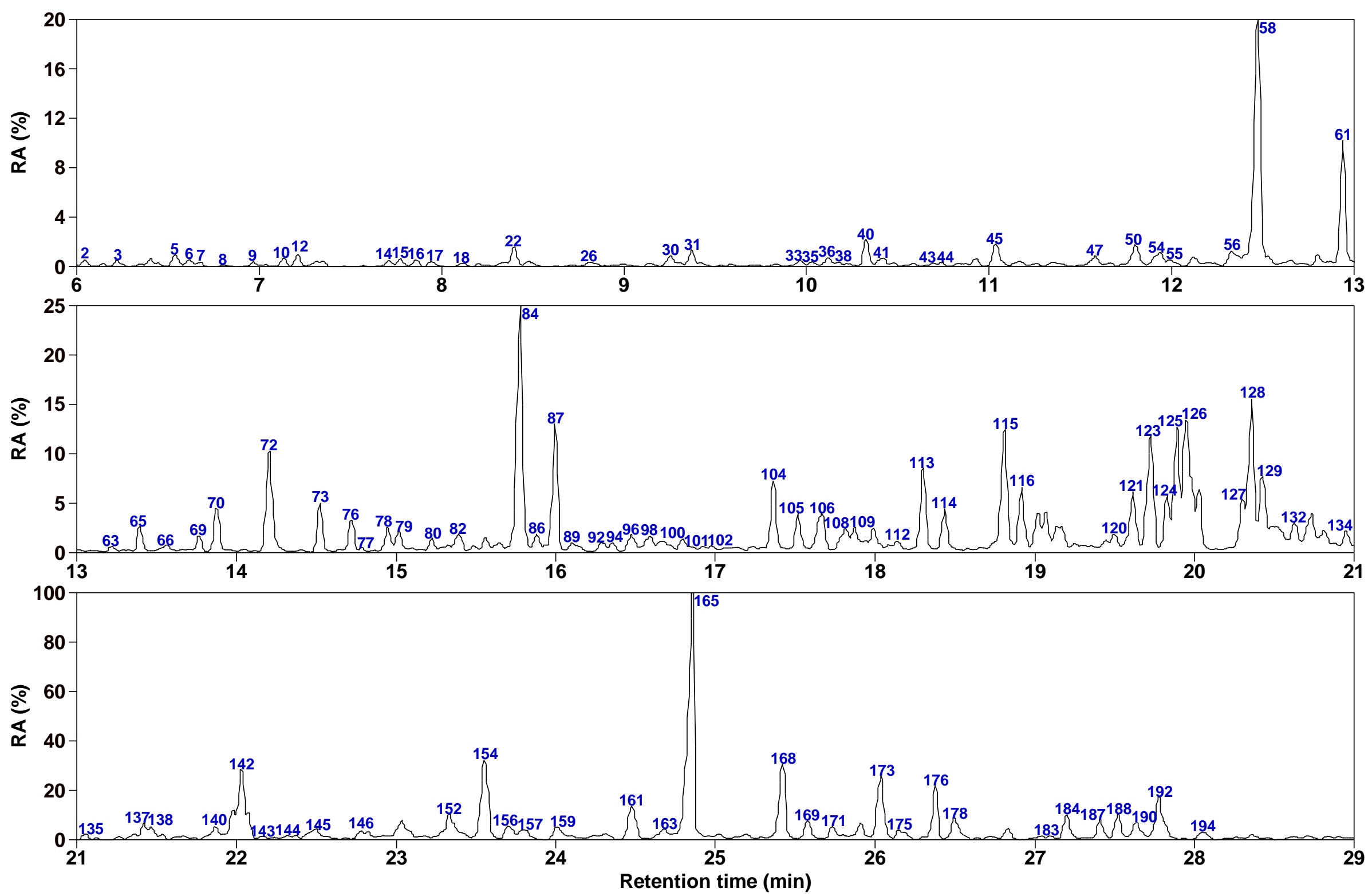

Figure S8. Total ion chromatogram of $\mathrm{SP}_{\mathrm{CHC}-\mathrm{F} \text {. }}$ 

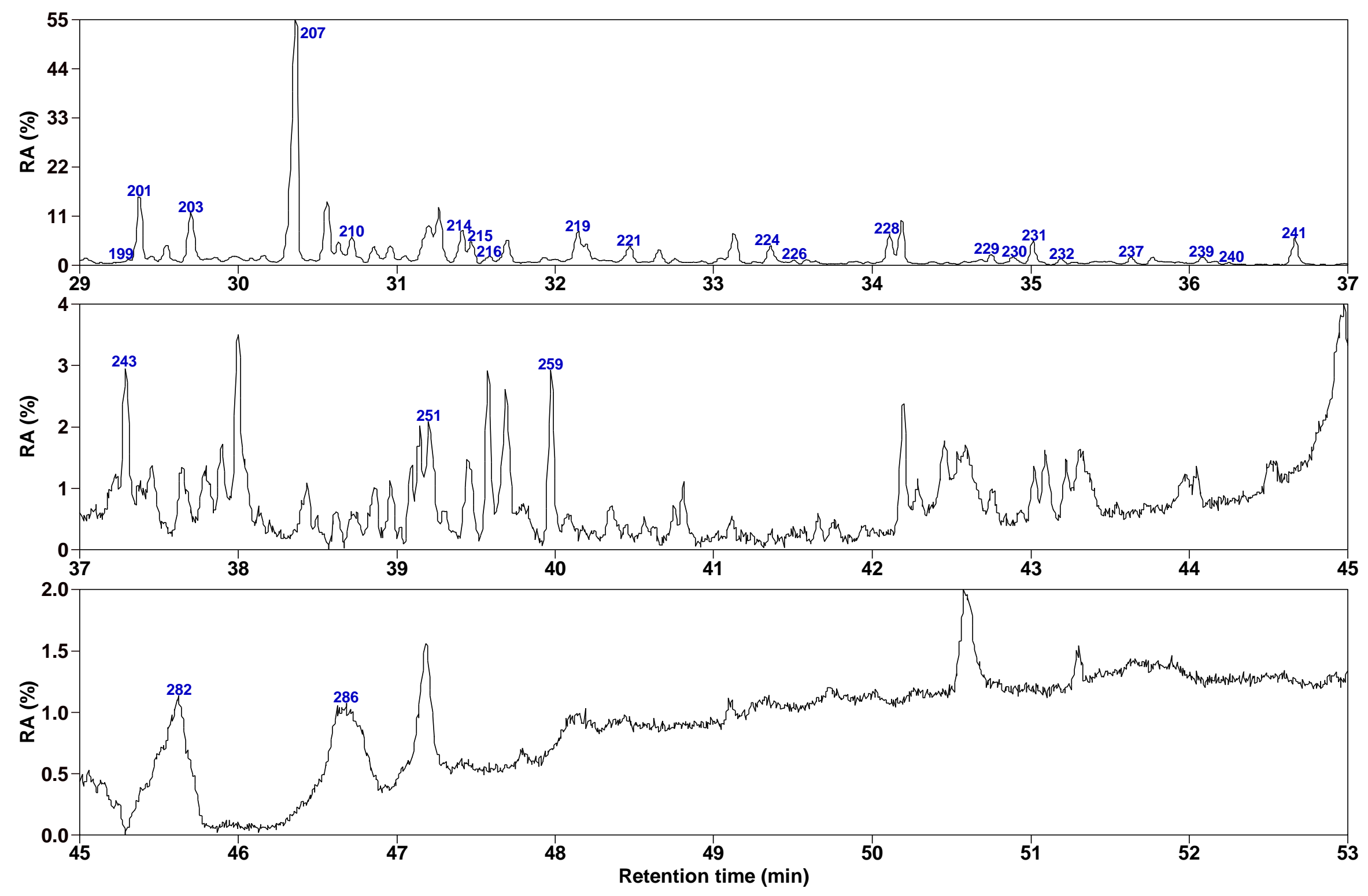

Figure S8. Total ion chromatogram of $\mathrm{SP}_{\mathrm{CHC}-\mathrm{F}}$ (continued). 
Table S1. NAs Detected in SPCHC-F

\begin{tabular}{ccccccccc}
\hline peak & NA & RC & peak & NA & RC & peak & NA & RC \\
\hline $\mathbf{4 1}$ & decane & 0.06 & $\mathbf{1 8 3}$ & hexadecane & 0.09 & $\mathbf{2 4 3}$ & heneicosane & 0.10 \\
$\mathbf{1 2 0}$ & tridecane & 0.10 & $\mathbf{1 9 9}$ & heptadecane & 0.08 & & & \\
$\mathbf{1 4 3}$ & tetradecane & 0.19 & $\mathbf{2 2 6}$ & nonadecane & 0.10 & & & \\
\hline
\end{tabular}

Table S2. ACHs ${ }^{\mathrm{I}}$ Detected in SP $\mathrm{SHC}_{\mathrm{L}}$ and $\mathrm{SP}_{\mathrm{CHC}-\mathrm{F}}$

\begin{tabular}{ccccccc}
\hline \multirow{2}{*}{ peak } & \multirow{2}{*}{$\mathrm{ACH}^{\mathrm{I}}$} & \multicolumn{2}{c}{$\mathrm{RC}$} & \multirow{2}{*}{ peak } & $\mathrm{ACH}^{\mathrm{I}}$ & \multirow{2}{*}{$\mathrm{RC}$ in $\mathrm{SP}_{\mathrm{CHC}-\mathrm{F}}$} \\
\cline { 3 - 5 } & & $\mathrm{SP}_{\mathrm{CHC}-\mathrm{L}}$ & $\mathrm{SP}_{\mathrm{CHC}-\mathrm{F}}$ & & & \\
\hline $\mathbf{1 2}$ & ethylcyclohexane & 0.18 & 0.08 & $\mathbf{7 7}$ & pentylcyclohexane & 0.04 \\
$\mathbf{2 2}$ & propylcyclohexane & & 0.12 & $\mathbf{1 0 9}$ & hexylcyclohexane & 0.11 \\
$\mathbf{3 1}$ & butylcyclohexane & 0.04 & 0.10 & & & \\
\hline
\end{tabular}

Table S3. DMCHs Detected in SP $\mathrm{CHC}_{\mathrm{L}}$ and SP $\mathrm{SHC}_{\mathrm{CH}}$

\begin{tabular}{cccccccc}
\hline \multirow{2}{*}{ peak } & \multirow{2}{*}{$\mathrm{DMCH}$} & \multicolumn{2}{c}{$\mathrm{RC}$} & \multirow{2}{*}{ peak } & $\mathrm{DMCH}$ & \multicolumn{2}{c}{$\mathrm{RC}$} \\
\cline { 3 - 5 } & & $\mathrm{SP}_{\mathrm{CHC}-\mathrm{L}}$ & $\mathrm{SP}_{\mathrm{CHC}-\mathrm{F}}$ & & & $\mathrm{SP}_{\mathrm{CHC}-\mathrm{L}}$ & $\mathrm{SP}_{\mathrm{CHC}-\mathrm{F}}$ \\
\hline $\mathbf{3}$ & $1,3-\mathrm{DMCH}$ & 0.05 & 0.03 & $\mathbf{8}$ & $(1 S, 4 S)-1,4-\mathrm{DMCH}$ & & 0.01 \\
$\mathbf{6}$ & $1,2-\mathrm{DMCH}$ & 0.02 & 0.03 & $\mathbf{9}$ & $(1 R, 3 S)-1,3-\mathrm{DMCH}$ & & 0.03 \\
$\mathbf{7}$ & $1,4-\mathrm{DMCH}$ & 0.02 & 0.01 & $\mathbf{1 0}$ & $(1 R, 2 S)-1,2-\mathrm{DMCH}$ & 0.01 & 0.05 \\
\hline
\end{tabular}

Table S4. EMCHs Detected in SPCHC-L and SPCHC-F

\begin{tabular}{|c|c|c|c|c|c|c|}
\hline \multirow{2}{*}{ peak } & \multirow{2}{*}{$\mathrm{EMCH}$} & \multicolumn{2}{|c|}{$\mathrm{RC}$} & \multirow{2}{*}{ peak } & \multirow{2}{*}{$\mathrm{EMCH}$} & \multirow{2}{*}{$\begin{array}{l}\mathrm{RC} \text { in } \\
\mathrm{SP}_{\mathrm{CHC}-\mathrm{F}}\end{array}$} \\
\hline & & $\mathrm{SP}_{\mathrm{CHC}-\mathrm{L}}$ & $\mathrm{SP}_{\mathrm{CHC}-\mathrm{F}}$ & & & \\
\hline 14 & 1-ethyl-2-methylcyclohexane & & 0.03 & 26 & 1-ethyl-3-methylcyclohexane & 0.03 \\
\hline 21 & 1-ethyl-4-methylcyclohexane & 0.02 & & & & \\
\hline
\end{tabular}

Table S5. MPCHs Detected in SPCHC-F

\begin{tabular}{cccccc}
\hline peak & MPCH & RC & peak & MPCH & RC \\
\hline 35 & 1-methyl-3-propylcyclohexane & 0.03 & $\mathbf{4 4}$ & 1-methyl-2-propylcyclohexane & 0.02 \\
\hline
\end{tabular}

Table S6. IPMCHs Detected in SPCHC-L and SPCHC-F

\begin{tabular}{cccc}
\hline \multirow{2}{*}{ peak } & \multirow{2}{*}{ IPMCH } & \multicolumn{2}{c}{$\mathrm{RC}$} \\
\cline { 3 - 4 } & & $\mathrm{SP}_{\text {CHC-L }}$ & $\mathrm{SP}_{\text {CHC-F }}$ \\
\hline $\mathbf{4 7}$ & 1-isopropyl-4-methylcyclohexane & & 0.08 \\
$\mathbf{6 4}$ & 1-isopropyl-2-methylcyclohexane & 0.01 & \\
\hline
\end{tabular}

Table S7. TMCHs detected in SPCHC-L and SP $\mathrm{SHC}_{\mathrm{CH}}$

\begin{tabular}{cccccc}
\hline peak & TMCH & RC in SP $_{\text {CHC-L }}$ & peak & TMCH & RC in SP $_{\text {CHC-F }}$ \\
\hline $\mathbf{1 3}$ & $1,3,5-\mathrm{TMCH}$ & 0.03 & $\mathbf{1 8}$ & $1,2,4-\mathrm{TMCH}$ & 0.02 \\
\hline
\end{tabular}

Table S8. NSPCs Detected in SPCHC-L and SPCHC-F

\begin{tabular}{|c|c|c|c|c|c|c|c|}
\hline \multirow{2}{*}{ peak } & \multirow{2}{*}{ NSPC } & \multicolumn{2}{|c|}{$\mathrm{RC}$} & \multirow{2}{*}{ peak } & \multirow{2}{*}{ NSPC } & \multicolumn{2}{|c|}{$\mathrm{RC}$} \\
\hline & & $\mathrm{SP}_{\mathrm{CHC}-\mathrm{L}}$ & $\mathrm{SP}_{\mathrm{CHC}-\mathrm{F}}$ & & & $\mathrm{SP}_{\mathrm{CHC}-\mathrm{L}}$ & $\mathrm{SP}_{\mathrm{CHC}-\mathrm{F}}$ \\
\hline 30 & $(3 \mathrm{a} S, 7 \mathrm{a} S)$-perhydroindane & 0.07 & & 70 & perhydroazulene & & 0.43 \\
\hline 36 & $(3 \mathrm{a} R, 7 \mathrm{a} R)$-perhydroindane & 0.15 & 0.05 & 113 & trans-perhydroacenaphthene & & 0.94 \\
\hline 40 & $(3 \mathrm{a} R, 7 \mathrm{a} S)$-perhydroindane & 0.07 & 0.19 & 114 & trans-perhydroas-indacene & 0.43 & 0.47 \\
\hline 58 & trans-decalin & & 1.88 & 123 & cis-perhydroas-indacene & 1.14 & 1.51 \\
\hline 61 & cis-decalin & 8.94 & 0.80 & 124 & cis-perhydroacenaphthene & & 0.41 \\
\hline
\end{tabular}


Table S8. NSPCs Detected in SPCHC-L and SPCHC-F (Continued)

\begin{tabular}{lccc}
\hline & & \multicolumn{2}{c}{$\mathrm{RC}$} \\
\cline { 3 - 4 } peak & NSPC & $\mathrm{SP}_{\text {CHC-L }}$ & $\mathrm{SP}_{\text {CHC-F }}$ \\
\hline $\mathbf{1 2 6}$ & $\mathrm{bi}(\mathrm{cyclohexane})$ & & 0.82 \\
$\mathbf{1 6 1}$ & $(4 \mathrm{a} R, 8 \mathrm{a} S, 9 \mathrm{a} R, 10 \mathrm{a} S)$-perhydroanthracene & & 2.07 \\
$\mathbf{1 6 2}$ & $(4 \mathrm{a} R, 8 \mathrm{a} S, 9 \mathrm{a} S, 10 \mathrm{a} R)$-perhydroanthracene & 0.94 & \\
$\mathbf{1 6 5}$ & $(4 \mathrm{a} R, 8 \mathrm{a} R, 9 \mathrm{a} S, 10 \mathrm{a} S)$-perhydroanthracene & 13.29 & 4.34 \\
$\mathbf{1 6 8}$ & $(4 \mathrm{a} R, 8 \mathrm{a} R, 9 \mathrm{a} S, 10 \mathrm{a} S)$-perhydrophenanthrene & 3.67 & 1.36 \\
$\mathbf{1 7 3}$ & $(4 \mathrm{a} R, 8 \mathrm{a} S, 9 \mathrm{a} S, 10 \mathrm{a} R)$-perhydrophenanthrene & 3.27 & 1.10 \\
$\mathbf{2 0 1}$ & perhydropyrene & 0.98 & 1.68 \\
$\mathbf{2 1 5}$ & perhydrofluoranthene & 0.18 & 0.39 \\
$\mathbf{2 3 2}$ & perhydrotetracene & 0.33 & 0.19 \\
$\mathbf{2 4 0}$ & perhydrotetraphene & 0.16 & 0.07 \\
$\mathbf{2 2 9}$ & perhydrochrysene & 0.38 & 0.20 \\
$\mathbf{2 0 3}$ & perhydrophenanthrene & 1.08 & \\
$\mathbf{2 3 5}$ & perhydrotriphenylene & 0.10 & 1.47 \\
\hline
\end{tabular}

Table S9. ADs detected in $\mathrm{SP}_{\mathrm{CHC}-\mathrm{L}}$ and $\mathrm{SP}_{\mathrm{CHC}-\mathrm{F}}$.

\begin{tabular}{cccc}
\hline & $\mathrm{AD}$ & \multicolumn{2}{c}{$\mathrm{RC}$} \\
\cline { 3 - 4 } peak & $(1 S, 4 \mathrm{a} R, 8 \mathrm{a} R)$-2-methyldecalin & $\mathrm{SP}_{\mathrm{CHC}-\mathrm{L}}$ & $\mathrm{SP}_{\mathrm{CHC}-\mathrm{F}}$ \\
\hline $\mathbf{7 2}$ & $(1 R, 4 \mathrm{a} R, 8 \mathrm{a} R)$-2-methyldecalin & 0.61 & 1.25 \\
$\mathbf{7 3}$ & $(1 R, 4 \mathrm{a} S, 8 \mathrm{a} R)$-2-methyldecalin & & 0.46 \\
$\mathbf{7 6}$ & $(1 S, 4 \mathrm{a} R, 8 \mathrm{a} R)$-1-methyldecalin & 0.35 & 0.32 \\
$\mathbf{7 9}$ & $(1 R, 4 \mathrm{a} R, 8 \mathrm{a} R)$-1-methyldecalin & 0.16 & 0.33 \\
$\mathbf{8 0}$ & $(1 R, 4 \mathrm{a} S, 8 \mathrm{a} R)$-1-methyldecalin & 0.20 & 0.13 \\
$\mathbf{8 2}$ & $(1 S, 4 \mathrm{a} R, 8 \mathrm{a} R)$-2-ethyldecalin & & 0.23 \\
$\mathbf{1 0 6}$ & $(1 R, 4 \mathrm{a} R, 8 \mathrm{a} R)$-2-ethyldecalin & 0.02 & 0.63 \\
$\mathbf{1 0 8}$ & $1-\mathrm{ethyldecalin}$ & & 0.21 \\
$\mathbf{1 1 2}$ & 2-butyldecalin & & 0.11 \\
$\mathbf{1 5 2}$ & & & 0.40 \\
\hline
\end{tabular}

Table S10. DMDs Detected in SPCHC-L and SP CHC-F $_{\text {CHC }}$

\begin{tabular}{|c|c|c|c|c|c|c|c|}
\hline \multirow{2}{*}{ peak } & \multirow{2}{*}{ DMD } & \multicolumn{2}{|c|}{$\mathrm{RC}$} & \multirow{2}{*}{ peak } & \multirow{2}{*}{ DMD } & \multicolumn{2}{|c|}{$\mathrm{RC}$} \\
\hline & & $\mathrm{SP}_{\mathrm{CHC}-\mathrm{L}}$ & $\mathrm{SP}_{\mathrm{CHC}-\mathrm{F}}$ & & & $\mathrm{SP}_{\mathrm{CHC}-\mathrm{L}}$ & $\mathrm{SP}_{\mathrm{CHC}-\mathrm{F}}$ \\
\hline 86 & 2,6-DMD & & 0.16 & 94 & 2,3-DMD & 0.04 & 0.08 \\
\hline 89 & 1,5-DMD & & 0.14 & 98 & 1,2-DMD & 0.03 & 0.17 \\
\hline 90 & 1,3-DMD & 0.04 & & 101 & 1,4-DMD & 0.01 & 0.04 \\
\hline 92 & 1,6-DMD & & 0.10 & & & & \\
\hline
\end{tabular}

Table S11. MPHPs Detected in SP $\mathrm{CHC}_{\mathrm{L}}$ and SP $\mathrm{PHC}_{\mathrm{CH}}$

\begin{tabular}{|c|c|c|c|c|c|c|}
\hline \multirow{2}{*}{ peak } & \multirow{2}{*}{ MPHP } & \multicolumn{2}{|c|}{$\mathrm{RC}$} & \multirow{2}{*}{ peak } & \multirow{2}{*}{ МPHP } & \multirow{2}{*}{$\mathrm{RC}$ in $\mathrm{SP}_{\mathrm{CHC}-\mathrm{F}}$} \\
\hline & & $\mathrm{SP}_{\mathrm{CHC}-\mathrm{L}}$ & $\mathrm{SP}_{\mathrm{CHC}-\mathrm{F}}$ & & & \\
\hline 175 & 2-MPHP & 0.67 & 0.15 & 178 & 1-MPHP & 1.28 \\
\hline
\end{tabular}

Table S12. OCs detected in SPCHC-F

\begin{tabular}{cccccc}
\hline peak & OC & RC & peak & OC & RC \\
\hline $\mathbf{5 4}$ & 5-methylperhydroindane & 0.12 & $\mathbf{1 4 0}$ & dicyclohexylmethane & 0.49 \\
\hline
\end{tabular}


Table S13. ASCHs Detected in SPCHC-L and SPCHC-F

\begin{tabular}{|c|c|c|c|c|c|}
\hline \multirow{2}{*}{ peak } & \multirow{2}{*}{\multicolumn{3}{|c|}{$\mathrm{ASCH}$}} & \multicolumn{2}{|l|}{$\mathrm{RC}$} \\
\hline & & & & \multirow{3}{*}{$\frac{\mathrm{SP}_{\mathrm{CHC}-\mathrm{L}}}{0.82}$} & $\mathrm{SP}_{\mathrm{CHC}-\mathrm{F}}$ \\
\hline 45 & \multirow{2}{*}{\multicolumn{3}{|c|}{$\begin{array}{l}\text { 1-butylcyclohex-1-ene } \\
\text { 1-isopropyl-4-methylcyclohex-1-ene }\end{array}$}} & & 0.19 \\
\hline 55 & & & & & 0.02 \\
\hline \multicolumn{6}{|c|}{ Table S14. NSCAs Detected in SPNCHC } \\
\hline peak & NSCA & $\mathrm{RC}$ & peak & NSCA & $\mathrm{RC}$ \\
\hline 118 & naphthalene & 29.41 & 310 & tetraphene & 1.36 \\
\hline 193 & biphenylene & 3.73 & 320 & benzo[e]pyrene & 0.53 \\
\hline 244 & anthracene & 12.25 & 321 & benz $[e]$ acephenanthrylene & 0.64 \\
\hline 245 & phenanthrene & 1.91 & 322 & benzo $[k]$ fluoranthene & 0.38 \\
\hline 279 & fluoranthene & 7.32 & 324 & perylene & 0.79 \\
\hline 283 & pyrene & 5.95 & 325 & benzo[pqr]tetraphene & 1.28 \\
\hline 305 & benzo $[c]$ phenanthrene & 0.44 & 326 & benzo[j]fluoranthene & 0.37 \\
\hline 309 & triphenylene & 2.03 & & & \\
\hline
\end{tabular}

Table S15. PAs Detected in SPNCHC and SPCHC-L

\begin{tabular}{|c|c|c|c|c|c|c|}
\hline \multirow{2}{*}{ peak } & \multirow{2}{*}{ PA } & \multicolumn{2}{|c|}{$\mathrm{RC}$} & \multirow{2}{*}{ peak } & \multirow{2}{*}{ PA } & \multirow{2}{*}{$\mathrm{RC}$ in $\mathrm{SP}_{\mathrm{NCHC}}$} \\
\hline & & $\mathrm{SP}_{\mathrm{NCHC}}$ & $\mathrm{SP}_{\mathrm{CHC}-\mathrm{L}}$ & & & \\
\hline 141 & biphenyl & 0.63 & 0.29 & 302 & terphenyl & 0.04 \\
\hline 254 & 1-phenylnaphthalene & 0.19 & & 318 & 2,2'-binaphthalene & 0.04 \\
\hline 268 & 2-phenylnaphthalene & 0.30 & & & & \\
\hline
\end{tabular}

Table S16. SMCCAs Detected in SPNCHC, SPCHC-L, and SPCHC-F

\begin{tabular}{ccccc}
\hline \multirow{2}{*}{ peak } & SMCCA & \multicolumn{3}{c}{$\mathrm{RC}$} \\
\cline { 3 - 4 } $\mathbf{7 1}$ & indene & 3.50 & & $\mathrm{SP}_{\mathrm{CHC}-\mathrm{L}}$ \\
$\mathbf{1 4 6}$ & diphenylmethane & & & 0.74 \\
$\mathbf{1 8 4}$ & fluorene & & 1.13 & 1.36 \\
$\mathbf{2 1 7}$ & phenalene & 0.07 & & \\
$\mathbf{2 6 3}$ & cyclopenta[def]phenanthrene & 1.44 & \\
$\mathbf{2 9 3}$ & benzo[b]fluorene & 0.66 & \\
$\mathbf{2 9 4}$ & benzo[a]fluorene & 0.46 & \\
$\mathbf{3 1 9}$ & $9 H$-cyclopenta $[a]$ pyrene & 0.49 & \\
$\mathbf{3 2 7}$ & $13 H$-dibenzo $[a, h]$ fluorene & 0.11 & \\
\hline
\end{tabular}

Table S17. ABs Detected in SPNCHC, SPCHC-L, and SPCHC-F

\begin{tabular}{|c|c|c|c|c|c|c|c|}
\hline \multirow{2}{*}{ peak } & \multirow{2}{*}{$\mathrm{AB}$} & \multicolumn{3}{|c|}{$\mathrm{RC}$} & \multirow{2}{*}{ peak } & \multirow{2}{*}{$\mathrm{AB}$} & \multirow{2}{*}{$\mathrm{RC}$ in $\mathrm{SP}_{\mathrm{CHC}-\mathrm{L}}$} \\
\hline & & $\mathrm{SP}_{\mathrm{NCHC}}$ & SP $_{\text {CHC-L }}$ & SP $_{\text {CHC-F }}$ & & & \\
\hline 2 & toluene & 0.32 & 0.66 & 0.03 & 29 & cumene & 0.04 \\
\hline 15 & ethylbenzene & & 1.21 & 0.08 & 34 & propylbenzene & 0.03 \\
\hline
\end{tabular}

Table S18. DABs Detected in SPNCHC, SPCHC-L, and SPCHC-F $_{\text {H }}$

\begin{tabular}{ccccccccc}
\hline \multirow{2}{*}{ peak } & \multirow{2}{*}{$\mathrm{DAB}$} & \multicolumn{3}{c}{$\mathrm{RC}$} & \multirow{2}{*}{ peak } & \multirow{2}{*}{$\mathrm{DAB}$} & \multicolumn{2}{c}{$\mathrm{RC}$} \\
\cline { 3 - 8 } & & $\mathrm{SP}_{\mathrm{NCHC}}$ & $\mathrm{SP}_{\mathrm{CHC}-\mathrm{L}}$ & $\mathrm{SP}_{\mathrm{CHC}-\mathrm{F}}$ & & & $\mathrm{SP}_{\mathrm{CHC}-\mathrm{L}}$ & $\mathrm{SP}_{\mathrm{CHC}-\mathrm{F}}$ \\
\hline $\mathbf{1 7}$ & $p$-xylene & & 0.41 & 0.06 & $\mathbf{3 8}$ & 1-ethyl-2-toluene & 0.12 & 0.01 \\
$\mathbf{2 3}$ & $m$-xylene & 0.32 & 0.17 & & $\mathbf{3 9}$ & 1-ethyl-4-toluene & 0.03 & \\
\hline
\end{tabular}




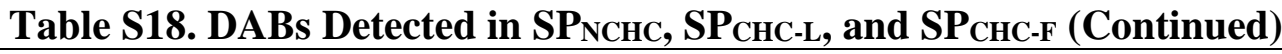

\begin{tabular}{ccccccc}
\hline \multirow{2}{*}{ peak } & \multirow{2}{*}{$\mathrm{DAB}$} & \multicolumn{2}{c}{$\mathrm{RC}$} & \multirow{2}{*}{ peak } & $\mathrm{DAB}$ & $\mathrm{RC}$ \\
\cline { 2 - 5 } & & $\mathrm{SP}_{\mathrm{NCHC}}$ & $\mathrm{SP}_{\mathrm{CHC}-\mathrm{F}}$ & & & $\mathrm{SP}_{\mathrm{CHC}-\mathrm{L}}$ \\
\hline $\mathbf{2 8}$ & $\begin{array}{c}o \text {-xylene } \\
\mathbf{3 3}\end{array}$ & 0.09 & & $\mathbf{7 4}$ & $o$-cymene & 0.01 \\
\hline
\end{tabular}

Table S19. TABs Detected in SPNCHC, SPCHC-L, and SPCHC-F

\begin{tabular}{ccccccccc}
\hline \multirow{2}{*}{ peak } & \multirow{2}{*}{$\mathrm{TAB}$} & \multicolumn{3}{c}{$\mathrm{RC}$} & \multirow{2}{*}{ peak } & TAB & \multicolumn{2}{c}{$\mathrm{RC}$} \\
\cline { 3 - 8 } & & $\mathrm{SP}_{\mathrm{NCHC}}$ & $\mathrm{SP}_{\mathrm{CHC}-\mathrm{L}}$ & $\mathrm{SP}_{\mathrm{CHC}-\mathrm{F}}$ & & $\mathrm{SP}_{\mathrm{CHC}-\mathrm{F}}$ \\
\hline $\mathbf{4 3}$ & mesitylene & 0.06 & 0.07 & 0.01 & $\mathbf{6 2}$ & hemimellitene & 0.03 & \\
$\mathbf{5 3}$ & pseudocumene & 0.17 & 0.03 & & $\mathbf{6 7}$ & 1-ethyl-2,3-xylene & 0.01 & 0.02 \\
$\mathbf{6 0}$ & 2-ethyl-1,4-xylene & & 0.02 & & & & & \\
\hline
\end{tabular}

Table S20. TMBs Detected in SPNCHC

\begin{tabular}{ccccccccc}
\hline peak & TMB & RC & peak & TMB & RC & peak & TMB & RC \\
\hline $\mathbf{9 5}$ & durene & 0.01 & $\mathbf{9 7}$ & isodurene & 0.01 & $\mathbf{9 9}$ & prehenitene & 0.01 \\
\hline
\end{tabular}

Table S21. AAs Detected in SPCHC-L and SPCHC-F

\begin{tabular}{cccccccc}
\hline \multirow{2}{*}{ peak } & \multirow{2}{*}{$\mathrm{AA}$} & \multicolumn{2}{c}{$\mathrm{RC}$} & \multirow{2}{*}{ pa } & \multicolumn{2}{c}{$\mathrm{RC}$} \\
\cline { 3 - 6 } \cline { 6 - 7 } & & $\mathrm{SP}_{\mathrm{CHC}-\mathrm{L}}$ & $\mathrm{SP}_{\mathrm{CHC}-\mathrm{F}}$ & & & $\mathrm{SP}_{\mathrm{CHC}-\mathrm{L}}$ & $\mathrm{SP}_{\mathrm{CHC}-\mathrm{F}}$ \\
\hline $\mathbf{2 7}$ & styrene & 0.16 & & $\mathbf{6 9}$ & isobutenylbenzene & & 0.15 \\
$\mathbf{4 9}$ & prop-1-en-2-ylbenzene & 0.01 & & $\mathbf{8 3}$ & but-1-en-1-ylbenzene & 0.01 & \\
$\mathbf{5 6}$ & allylbenzene & & 0.12 & $\mathbf{1 7 9}$ & 2-vinylnaphthalene & 0.03 \\
$\mathbf{6 8}$ & 2-vinyl-1,4-xylene & 0.02 & & $\mathbf{1 8 5}$ & 1-vinylnaphthalene & 0.15 \\
\hline
\end{tabular}

Table S22. MIs ${ }^{\mathrm{I}}$ Detected in SPNCHC

\begin{tabular}{cccccc}
\hline peak & MI $^{\mathrm{I}}$ & $\mathrm{RC}$ & peak & MI $^{\mathrm{I}}$ & $\mathrm{RC}$ \\
\hline $\mathbf{1 0 7}$ & 2-methylindene & 0.07 & $\mathbf{1 1 0}$ & 1-methylindene & 0.08 \\
\hline
\end{tabular}

Table S23. MSBPs Detected in SPNCHC and SPCHC-L

\begin{tabular}{cccccccc}
\hline \multirow{2}{*}{ peak } & \multirow{2}{*}{ MSBP } & \multicolumn{2}{c}{$\mathrm{RC}$} & \multirow{2}{*}{ MSBP } & \multicolumn{2}{c}{$\mathrm{RC}$} \\
\cline { 3 - 7 } & & $\mathrm{SP}_{\mathrm{NCHC}}$ & $\mathrm{SP}_{\mathrm{CHC}-\mathrm{L}}$ & & & $\mathrm{SP}_{\mathrm{NCHC}}$ & $\mathrm{SP}_{\mathrm{CHC}-\mathrm{L}}$ \\
\hline $\mathbf{1 6 3}$ & 3-methylbiphenyl & & 0.28 & $\mathbf{2 2 0}$ & 3,3'-dimethylbiphenyl & 0.02 & 0.49 \\
$\mathbf{1 9 6}$ & 4-methylbiphenyl & 0.16 & & $\mathbf{2 4 9}$ & 3,3',4,4'-tetramethylbiphenyl & 0.01 & \\
$\mathbf{1 9 8}$ & 2-methylbiphenyl & 0.07 & & & & & \\
\hline
\end{tabular}

Table S24. MFs ${ }^{I}$ Detected in SP $_{\text {NCHC }}$ and SPCHC-L

\begin{tabular}{cccccccccc}
\hline \multirow{2}{*}{ peak } & \multirow{2}{*}{$\mathrm{MF}^{\mathrm{I}}$} & \multicolumn{2}{c}{$\mathrm{RC}$} & \multirow{2}{*}{ peak } & $\mathrm{MF}^{\mathrm{I}}$ & \multirow{2}{*}{$\mathrm{RC}$ in $\mathrm{SP}_{\mathrm{NCHC}}$} & peak & $\mathrm{MF}^{\mathrm{I}}$ & \multirow{2}{*}{$\mathrm{RC}$ in $\mathrm{SP}_{\mathrm{NCHC}}$} \\
\cline { 3 - 7 } & & $\mathrm{SP}_{\mathrm{NCHC}}$ & $\mathrm{SP}_{\mathrm{CHC}-\mathrm{L}}$ & & & & & \\
$\mathbf{2 0 4}$ & $1-\mathrm{MF}^{\mathrm{I}}$ & & 0.06 & $\mathbf{2 3 4}$ & $2-\mathrm{MF}^{\mathrm{I}}$ & 0.20 & $\mathbf{2 3 8}$ & $9-\mathrm{MF}^{\mathrm{I}}$ & 0.06 \\
$\mathbf{2 1 8}$ & $4-\mathrm{MF}^{\mathrm{I}}$ & 3.86 & & $\mathbf{2 3 6}$ & $3-\mathrm{MF}^{\mathrm{I}}$ & 0.08 & & & \\
\hline
\end{tabular}

Table S25. ANs ${ }^{\mathrm{I}}$ Detected in SPNCHC

\begin{tabular}{cccccc}
\hline peak & AN $^{\mathrm{I}}$ & $\mathrm{RC}$ & peak & $\mathrm{AN}^{\mathrm{I}}$ & $\mathrm{RC}$ \\
\hline $\mathbf{1 4 8}$ & 2-methylnaphthalene & 2.93 & $\mathbf{1 7 4}$ & 1-ethylnaphthalene & 0.01 \\
$\mathbf{1 5 3}$ & 1-methylnaphthalene & 1.19 & & & \\
\hline
\end{tabular}


Table S26. DMNs Detected in SP $\mathbf{N C H C}_{\mathrm{N}}$

\begin{tabular}{ccccccccc}
\hline peak & DMN & RC & peak & DMN & RC & peak & DMN & RC \\
\hline $\mathbf{1 7 7}$ & $2,6-D M N$ & 0.34 & $\mathbf{1 8 2}$ & $1,3-\mathrm{DMN}$ & 0.17 & $\mathbf{1 9 1}$ & $1,4-\mathrm{DMN}$ & 0.05 \\
$\mathbf{1 8 1}$ & $1,6-\mathrm{DMN}$ & 0.28 & $\mathbf{1 8 9}$ & $1,2-\mathrm{DMN}$ & 0.10 & $\mathbf{1 9 5}$ & $2,3-\mathrm{DMN}$ & 0.04 \\
\hline
\end{tabular}

Table S27. TMNs Detected in SP NCHC

\begin{tabular}{ccccccccc}
\hline peak & TMN & RC & peak & TMN & RC & peak & TMN & RC \\
\hline $\mathbf{2 0 6}$ & $1,4,5-\mathrm{TMN}$ & 0.03 & $\mathbf{2 1 1}$ & $1,6,7-\mathrm{TMN}$ & 0.04 & $\mathbf{2 1 3}$ & $1,2,3-\mathrm{TMN}$ & 0.02 \\
$\mathbf{2 0 8}$ & $2,3,6-\mathrm{TMN}$ & 0.04 & $\mathbf{2 1 2}$ & $1,4,6-\mathrm{TMN}$ & 0.03 & & & \\
\hline
\end{tabular}

Table S28. MAs Detected in SPNCHC

\begin{tabular}{cccccc}
\hline peak & MA & RC & peak & MA & RC \\
\hline $\mathbf{2 6 1}$ & 2-MA & 0.50 & $\mathbf{2 6 2}$ & 1-MA & 0.23 \\
\hline
\end{tabular}

Table S29. DMAs Detected in SP $\mathbf{N C H C}_{\mathrm{N}}$

\begin{tabular}{ccccccccc}
\hline peak & DMA & RC & peak & DMA & RC & peak & DMA & RC \\
\hline $\mathbf{2 7 1}$ & 1,2-DMA & 0.04 & $\mathbf{2 7 2}$ & 1,7-DMA & 0.06 & $\mathbf{2 7 3}$ & 2,7-DMA & 0.03 \\
\hline \multicolumn{8}{c}{ Table S30. MPs detected in SPNCHC } \\
\hline peak & MP & RC & peak & MP & RC & peak & MP & RC \\
\hline $\mathbf{2 5 6}$ & 2-MP & 0.07 & $\mathbf{2 6 0}$ & 1-MP & 0.43 & $\mathbf{2 6 4}$ & 4-MP & 0.20 \\
\hline
\end{tabular}

Table S31. DMPs ${ }^{\mathrm{I}}$ Detected in SPNCHC

\begin{tabular}{cccccc}
\hline peak & DMP $^{\mathrm{I}}$ & RC & peak & DMP $^{\mathrm{I}}$ & RC \\
\hline $\mathbf{2 7 4}$ & $4,5-\mathrm{DMP}^{\mathrm{I}}$ & 0.07 & $\mathbf{2 7 6}$ & $9,10-\mathrm{DMP}^{\mathrm{I}}$ & 0.12 \\
$\mathbf{2 7 5}$ & $2,5-\mathrm{DMP}^{\mathrm{I}}$ & 0.06 & $\mathbf{2 7 7}$ & $1,9-\mathrm{DMP}^{\mathrm{I}}$ & 0.06 \\
\hline
\end{tabular}

Table S32. MFs ${ }^{\mathrm{II}}$ Detected in SPNCHC

\begin{tabular}{ccccccccc}
\hline peak & $\mathrm{MF}^{\mathrm{II}}$ & $\mathrm{RC}$ & peak & $\mathrm{MF}^{\mathrm{II}}$ & $\mathrm{RC}$ & peak & $\mathrm{MF}^{\mathrm{II}}$ & $\mathrm{RC}$ \\
\hline $\mathbf{2 9 0}$ & $3-\mathrm{MF}^{\mathrm{II}}$ & 0.04 & $\mathbf{2 9 2}$ & $2-\mathrm{MF}^{\mathrm{II}}$ & 0.07 & $\mathbf{2 9 5}$ & $1-\mathrm{MF}^{\mathrm{II}}$ & 0.19 \\
\hline
\end{tabular}

Table S33. MSPs ${ }^{\mathrm{I}}$ Detected in $\mathrm{SP}_{\mathrm{NCHC}}$

\begin{tabular}{cccccc}
\hline peak & MSP & RC & peak & MSP $^{I}$ & RC \\
\hline $\mathbf{2 9 6}$ & 1-methylpyrene & 0.21 & $\mathbf{2 9 9}$ & 2-methylpyrene & 0.11 \\
$\mathbf{2 9 7}$ & 8-methylpyrene & 0.12 & $\mathbf{3 0 0}$ & 7-methylpyrene & 0.08 \\
$\mathbf{2 9 8}$ & 4-methylpyrene & 0.12 & $\mathbf{3 0 3}$ & 1,3-dimethylpyrene & 0.06 \\
\hline
\end{tabular}

Table S34. MCs ${ }^{\mathrm{I}}$ Detected in SPNCHC

\begin{tabular}{ccccccccc}
\hline peak & $\mathrm{MC}^{\mathrm{I}}$ & $\mathrm{RC}$ & peak & $\mathrm{MC}^{\mathrm{I}}$ & $\mathrm{RC}$ & peak & $\mathrm{MC}^{\mathrm{I}}$ & $\mathrm{RC}$ \\
\hline $\mathbf{3 1 5}$ & $6-\mathrm{MC}^{\mathrm{I}}$ & 0.08 & $\mathbf{3 1 6}$ & $1-\mathrm{MC}$ & 0.24 & $\mathbf{3 1 7}$ & $2-\mathrm{MC}^{\mathrm{I}}$ & 0.13 \\
\hline
\end{tabular}

Table S35. NSHAs Detected in SPNCHC, SPCHC-L, and SPCHC-F

\begin{tabular}{cccc}
\hline \multirow{2}{*}{ peak } & NSHA & \multicolumn{2}{c}{$\mathrm{RC}$} \\
\cline { 3 - 4 } & & $\mathrm{SP}_{\mathrm{CHC}-\mathrm{L}}$ & $\mathrm{SP}_{\mathrm{CHC}-\mathrm{F}}$ \\
\hline $\mathbf{5 0}$ & indane & & 0.18 \\
$\mathbf{8 4}$ & tetralin & 23.95 & 12.85 \\
$\mathbf{1 4 2}$ & $2 a, 3,4,5$-yetrahydroacenaphthene & 2.60 & 4.89 \\
$\mathbf{1 5 4}$ & $(4 \mathrm{a} S, 9 \mathrm{a} R)-2,3,4,4 \mathrm{a}, 9,9 \mathrm{a}-$-hexahydrofluorene & 4.93 & 2.80 \\
$\mathbf{1 5 6}$ & $(4 \mathrm{a} R, 9 \mathrm{a} R)-2,3,4,4 \mathrm{a}, 9,9 \mathrm{a}-$-hexahydrofluorene & 0.83 & 0.33 \\
\hline
\end{tabular}


Table S35. NSHAs Detected in SP $\mathbf{N C H C}_{\mathrm{NC}} \mathrm{SP}_{\mathrm{CHC}-\mathrm{L}}$, and SPCHC-F (continued)

\begin{tabular}{|c|c|c|c|c|}
\hline \multirow{2}{*}{ peak } & \multirow{2}{*}{ NSHA } & \multicolumn{3}{|c|}{$\mathrm{RC}$} \\
\hline & & $\mathrm{SP}_{\mathrm{NCHC}}$ & $\mathrm{SP}_{\mathrm{CHC}-\mathrm{L}}$ & $\mathrm{SP}_{\mathrm{CHC}-\mathrm{F}}$ \\
\hline 186 & $(4 \mathrm{a} R, 10 \mathrm{a} R)-1,2,3,4,4 \mathrm{a}, 9,10,10 \mathrm{a}-$ octahydrophenanthrene & & 1.10 & \\
\hline 190 & $(4 \mathrm{a} R, 10 \mathrm{a} S)-1,2,3,4,4 \mathrm{a}, 9,10,10 \mathrm{a}-$-octahydrophenanthrene & & 1.02 & 1.03 \\
\hline 192 & $(4 \mathrm{a} R, 9 \mathrm{a} S)-1,2,3,4,4 \mathrm{a}, 9,9 \mathrm{a}, 10$-octahydroanthracene & & 2.56 & 1.42 \\
\hline 197 & acenaphthene & 0.20 & & 0.27 \\
\hline 207 & $1,2,3,4,5,6,7,8$-octahydroanthracene & & 7.28 & 4.15 \\
\hline 219 & $1,2,3,3 a, 3 a^{1}, 4,5,6,6 a, 10 \mathrm{~b}$-decahydrofluoranthene & & 1.46 & 0.90 \\
\hline 221 & $1,2,3,6 b, 7,8,9,10,10 a, 10 \mathrm{~b}$-decahydrofluoranthene & & 1.65 & 0.52 \\
\hline 224 & $1,2,3,3 a, 4,5,5 a, 6,7,8$-decahydropyrene & & 0.35 & 0.63 \\
\hline 228 & 1,2,3,4,5,6,7,8-octahydrophenanthrene & & 0.96 & 0.64 \\
\hline 233 & $1,2,3,10 b$-tetrahydrofluoranthene & & 0.09 & \\
\hline 237 & 2,3,4,6b,7,8,9,10,10a,11-decahydrobenzo[ $a]$ fluorene & & 0.26 & 0.21 \\
\hline 239 & $1,2,3,3 a, 4,5$-hexahydropyrene & & 0.09 & 0.20 \\
\hline 241 & $1,2,3,6,7,8$-hexahydropyrene & & 0.37 & 0.87 \\
\hline 311 & 3,4-dihydrocyclopenta $[c, d]$ pyrene & 0.11 & & \\
\hline 251 & $1,2,3,4,4 a, 7,8,9,10,11,12,12 a$-dodecahydrochrysene & & 0.15 & 0.07 \\
\hline 253 & 2,3,4,11-tetrahydrobenzo $[a]$ fluorene & & & \\
\hline 255 & $1,2,3,4,4 a, 5,7,8,9,10,12,12 a$-dodecahydrotetracene & 0.33 & & \\
\hline 257 & 1,2,3,4,7,7a,8,9,10,11,11a,12-dodecahydrotetraphene & 0.32 & & \\
\hline 259 & $1,2,3,4,5,6,7,8,9,10,11,12$-dodecahydrotriphenylene & & 0.37 & 0.13 \\
\hline 267 & $1,2,3,4,4 \mathrm{a}, 5,6,9,10,11,12,12 \mathrm{c}$-dodecahydrobenzo[ $c]$ phenanthrene & & 0.32 & \\
\hline 269 & 1,2,3,3a,3b,4,5,6,7,7a,9,10,11,12-tetradecahydrobenzoacephenanthrylene & & 0.11 & \\
\hline 270 & 1,2,3,3a,4,5,6,7,8,9,9a,10,11,12-tetradecahydroperylene & & 0.20 & \\
\hline
\end{tabular}

Table S36. MIs ${ }^{I I}$ Detected in SPCHC-L and SPCHC-F

\begin{tabular}{ccccccccc}
\hline peak & $\mathrm{MI}^{\mathrm{II}}$ & $\mathrm{RC}$ in $\mathrm{SP}_{\mathrm{CHC}-\mathrm{L}}$ & peak & $\mathrm{MI}^{\mathrm{II}}$ & $\mathrm{RC}$ in $\mathrm{SP}_{\mathrm{CHC}-\mathrm{F}}$ & peak & $\mathrm{MI}^{\mathrm{II}}$ & $\mathrm{RC}$ in $\mathrm{SP}_{\mathrm{CHC}-\mathrm{F}}$ \\
\hline $\mathbf{8 1}$ & $4-\mathrm{MI}^{\mathrm{II}}$ & 0.05 & $\mathbf{6 3}$ & $2-\mathrm{MI}^{\mathrm{II}}$ & 0.07 & $\mathbf{6 5}$ & $1-\mathrm{MI}^{\mathrm{II}}$ & 0.27 \\
\hline
\end{tabular}

Table S37. DMIs Detected in SPCHC-F

\begin{tabular}{cccccccccccc}
\hline peak & DMI & RC & peak & DMI & RC & peak & DMI & RC & peak & DMI & RC \\
\hline $\mathbf{9 6}$ & $1,6-$ DMI & 0.20 & $\mathbf{1 0 0}$ & $1,3-\mathrm{DMI}$ & 0.12 & $\mathbf{1 0 2}$ & $1,2-\mathrm{DMI}$ & 0.02 & $\mathbf{1 2 1}$ & $2,2-\mathrm{DMI}$ & 0.76 \\
\hline
\end{tabular}

Table S38. ATs Detected in SPCHC-L and SPCHC-F

\begin{tabular}{cccccccc}
\hline \multirow{2}{*}{ peak } & \multirow{2}{*}{$\mathrm{AT}$} & \multicolumn{3}{c}{$\mathrm{RC}$} & \multirow{2}{*}{ peak } & \multicolumn{2}{c}{$\mathrm{RC}$} \\
\cline { 3 - 6 } \cline { 6 - 8 } & & $\mathrm{SP}_{\mathrm{CHC}-\mathrm{L}}$ & $\mathrm{SP}_{\mathrm{CHC}-\mathrm{F}}$ & & & $\mathrm{SP}_{\mathrm{CHC}-\mathrm{L}}$ & $\mathrm{SP}_{\mathrm{CHC}-\mathrm{F}}$ \\
\hline $\mathbf{1 0 5}$ & 1-methyltetralin & 0.57 & 0.42 & $\mathbf{1 3 2}$ & 2-ethyltetralin & 0.15 & 0.26 \\
$\mathbf{1 1 5}$ & 5-methyltetralin & 1.26 & 0.88 & $\mathbf{1 3 7}$ & 5-ethyltetralin & 0.20 & 0.31 \\
$\mathbf{1 1 6}$ & 6-methyltetralin & & 0.67 & $\mathbf{1 3 8}$ & 6-ethyltetralin & 0.06 & 0.22 \\
$\mathbf{1 2 2}$ & 3-methyltetralin & 1.49 & & $\mathbf{1 7 6}$ & 6-butyltetralin & & 2.35 \\
$\mathbf{1 3 0}$ & 1-ethyltetralin & 0.02 & & & & & \\
\hline
\end{tabular}


Table S39. DMTs Detected in SPCHC-L and SPCHC-F

\begin{tabular}{cccccccc}
\hline \multirow{2}{*}{ peak } & \multirow{2}{*}{ compound } & \multicolumn{3}{c}{$\mathrm{RC}$} & \multirow{2}{*}{ peak } & compound & \multicolumn{2}{c}{$\mathrm{RC}$} \\
\cline { 3 - 6 } \cline { 6 - 7 } & & $\mathrm{SP}_{\mathrm{CHC}-\mathrm{L}}$ & $\mathrm{SP}_{\mathrm{CHC}-\mathrm{F}}$ & & & & \\
\hline $\mathbf{1 2 7}$ & $1,4-\mathrm{DMT}$ & 0.15 & 0.23 & $\mathbf{1 3 6}$ & $1,2-\mathrm{DMT}$ & 0.04 & \\
$\mathbf{1 2 9}$ & $2,3-\mathrm{DMT}$ & & 0.53 & $\mathbf{1 4 4}$ & $6,7-\mathrm{DMT}$ & 0.10 & 0.15 \\
$\mathbf{1 3 1}$ & $1,6-\mathrm{DMT}$ & 0.05 & & $\mathbf{1 4 5}$ & $5,6-\mathrm{DMT}$ & 0.02 & 0.13 \\
$\mathbf{1 3 4}$ & $2,6-\mathrm{DMT}$ & 0.07 & 0.16 & $\mathbf{1 5 1}$ & $1,3-\mathrm{DMT}$ & 0.04 & \\
$\mathbf{1 3 5}$ & $1,5-\mathrm{DMT}$ & 0.14 & 0.25 & $\mathbf{1 5 7}$ & $1,8-\mathrm{DMT}$ & & 0.50 \\
\hline
\end{tabular}

Table S40. AOHAs Detected in SPCHC-L and SPCHC-F

\begin{tabular}{cccc}
\hline \multirow{2}{*}{ peak } & \multirow{2}{*}{ AOHA } & \multicolumn{2}{c}{$\mathrm{RC}$} \\
\cline { 3 - 4 } & & SP $_{\text {CHC-L }}$ & SP $_{\text {CHC-F }}$ \\
\hline $\mathbf{2 1 0}$ & 1-methyl-1,2,3,4,5,6,7,8-octahydroanthracene & 0.22 & 0.44 \\
$\mathbf{2 1 4}$ & 2-methyl-1,2,3,4,5,6,7,8-octahydroanthracene & 0.27 & 0.62 \\
$\mathbf{2 1 6}$ & 1-methyl-1,2,3,4,5,6,7,8- octahydrophenanthrene & 0.09 & 0.24 \\
$\mathbf{2 2 3}$ & 9-ethyl-1,2,3,4,5,6,7,8-octahydroanthracene & 0.40 & \\
\hline
\end{tabular}

Table S41. OHAs Detected in SPCHC-L and SPCHC-F

\begin{tabular}{lcccccc}
\hline \multirow{2}{*}{ peak } & \multirow{2}{*}{ OHA } & \multirow{2}{*}{ RC in SP CHC-F peak } & \multirow{2}{*}{ OHA } & \multicolumn{2}{c}{ RC } \\
\cline { 5 - 8 } & & & & & SP $_{\text {CHC-L }}$ & SP $_{\text {CHC-F }}$ \\
\hline $\mathbf{1 8 8}$ & 3-(tert-butyl)-1,2-dihydronaphthalene & 1.21 & $\mathbf{2 3 1}$ & 5-phenyltetralin & 0.51 & 0.66 \\
\hline
\end{tabular}

Table S42. OAs Detected in SPNCHC, SPCHC-L, and SPCHC-F

\begin{tabular}{ccccc}
\hline \multirow{2}{*}{ peak } & OA & \multicolumn{3}{c}{$\mathrm{RC}$} \\
\cline { 3 - 5 } & & $\mathrm{SP}_{\mathrm{NCHC}}$ & $\mathrm{SP}_{\mathrm{CHC}-\mathrm{L}}$ & $\mathrm{SP}_{\mathrm{CHC}-\mathrm{F}}$ \\
\hline $\mathbf{2 4}$ & ethynylbenzene & 0.01 & \\
$\mathbf{1 2 8}$ & cyclohexylbenzene & & 0.27 & 1.26 \\
$\mathbf{2 3 0}$ & 2-(cyclohex-1-en-1-yl)naphthalene & & 0.21 & 0.24 \\
\hline
\end{tabular}

Table S43. CHs Detected in SPCHC-L and SPCHC-F

\begin{tabular}{|c|c|c|c|c|c|c|}
\hline \multirow{2}{*}{ peak } & \multirow{2}{*}{ compound } & \multirow{2}{*}{$\mathrm{RC}$ in $\mathrm{SP}_{\mathrm{CHC}-\mathrm{L}}$} & \multirow{2}{*}{ peak } & \multirow{2}{*}{ compound } & \multicolumn{2}{|c|}{$\mathrm{RC}$} \\
\hline & & & & & $\mathrm{SP}_{\mathrm{CHC}-\mathrm{L}}$ & $\mathrm{SP}_{\mathrm{CHC}-\mathrm{F}}$ \\
\hline 19 & $\mathrm{CH}$ & 0.07 & 125 & decalin-2-ol & 1.29 & 0.78 \\
\hline 32 & 3-methylcyclohexanol & 0.02 & & & & \\
\hline
\end{tabular}

Table S44. ACHs ${ }^{\mathrm{II}}$ Detected in SPCHC-L and SPCHC-F

\begin{tabular}{ccccccc}
\hline \multirow{2}{*}{ peak } & \multirow{2}{*}{$\mathrm{ACH}^{\mathrm{II}}$} & \multirow{2}{*}{$\mathrm{RC}$ in $\mathrm{SP}_{\mathrm{CHC}-\mathrm{F}}$} & peak & \multirow{2}{*}{$\mathrm{ACH}^{\mathrm{II}}$} & \multicolumn{2}{c}{$\mathrm{RC}$} \\
\cline { 6 - 7 } & & & & & $\mathrm{SP}_{\mathrm{CHC}-\mathrm{L}}$ & $\mathrm{SP}_{\mathrm{CHC}-\mathrm{F}}$ \\
\hline $\mathbf{5}$ & methoxycyclohexane & 0.08 & $\mathbf{1 6}$ & ethoxycyclohexane & 0.07 & 0.04 \\
\hline
\end{tabular}

Table S45. NSAs Detected in SPNCHC

\begin{tabular}{ccccccccc}
\hline peak & NSA & RC & peak & NSA & RC & peak & NSA & RC \\
\hline $\mathbf{4 6}$ & phenol & 0.12 & $\mathbf{2 2 2}$ & fluoren-1-ol & 0.39 & $\mathbf{3 2 3}$ & benzo[pqr]tetraphen-7-ol & 0.19 \\
$\mathbf{2 0 2}$ & naphth-1-ol & 0.02 & $\mathbf{2 8 9}$ & pyren-1-ol & 0.10 & & & \\
\hline
\end{tabular}

Table S46. MSPs ${ }^{\mathrm{II}}$ Detected in $\mathrm{SP}_{\mathrm{NCHC}}$

\begin{tabular}{ccccccccc}
\hline peak & MSP $^{\mathrm{II}}$ & $\mathrm{RC}$ & peak & MSP $^{\mathrm{II}}$ & $\mathrm{RC}$ & peak & MSP $^{\mathrm{II}}$ & $\mathrm{RC}$ \\
\hline $\mathbf{7 5}$ & $p$-cresol & 0.14 & $\mathbf{1 0 3}$ & 2,4-xylenol & 0.05 & $\mathbf{1 1 1}$ & 3,5 -xylenol & 0.03 \\
\hline
\end{tabular}


Table S47. ABFs Detected in SPNCHC, SPCHC-L, and SPCHC-F

\begin{tabular}{ccccc}
\hline \multirow{2}{*}{ peak } & ABF & \multicolumn{3}{c}{ RC } \\
\cline { 3 - 5 } & dibenzofuran & SP $_{\text {NCHC }}$ & SP $_{\text {CHC-L }}$ & SP $_{\text {CHC-F }}$ \\
\hline $\mathbf{1 6 9}$ & & & 0.99 & 1.03 \\
$\mathbf{2 8 5}$ & naphtho[2,1-b]benzofuran & 0.15 & & \\
$\mathbf{2 8 7}$ & naphtho[2,3-b]benzofuran & 0.20 & & \\
\hline
\end{tabular}

Table S48. BFs Detected in SPNCHC and SPCHC-L

\begin{tabular}{|c|c|c|c|c|c|c|}
\hline \multirow{2}{*}{ peak } & \multirow{2}{*}{ compound } & \multicolumn{2}{|c|}{$\mathrm{RC}$} & \multirow{2}{*}{ peak } & \multirow{2}{*}{ compound } & \multirow{2}{*}{$\mathrm{RC}$ in $\mathrm{SP}_{\mathrm{NCHC}}$} \\
\hline & & $\mathrm{SP}_{\mathrm{NCHC}}$ & $\mathrm{SP}_{\mathrm{CHC}-\mathrm{L}}$ & & & \\
\hline 57 & $\mathrm{BF}$ & 0.17 & 3.57 & 88 & 7-methylbenzofuran & 0.10 \\
\hline 85 & 2-methylbenzofuran & 0.04 & & 91 & 3-methylbenzofuran & 0.03 \\
\hline
\end{tabular}

Table S49. MDBFs Detected in SPNCHC, $\mathrm{SP}_{\mathrm{CHC}-\mathrm{L}}$, and SP $\mathrm{SHC}_{\mathrm{CH}}$

\begin{tabular}{ccccccccc}
\hline \multirow{2}{*}{ peak } & \multirow{2}{*}{ compound } & \multicolumn{3}{c}{$\mathrm{RC}$} & \multirow{2}{*}{ Peak } & compound & \multicolumn{2}{c}{$\mathrm{RC}$} \\
\cline { 3 - 5 } \cline { 7 - 9 } & & $\mathrm{SP}_{\mathrm{NCHC}}$ & $\mathrm{SP}_{\mathrm{CHC}-\mathrm{L}}$ & $\mathrm{SP}_{\mathrm{CHC}-\mathrm{F}}$ & & & $\mathrm{SP}_{\mathrm{NCHC}}$ & $\mathrm{SP}_{\mathrm{CHC}-\mathrm{L}}$ \\
\hline $\mathbf{1 9 4}$ & $4-\mathrm{MDBF}$ & & 0.18 & 0.53 & $\mathbf{2 2 7}$ & $5-\mathrm{MDBF}$ & 0.06 & \\
$\mathbf{2 2 5}$ & $1-\mathrm{MDBF}$ & 0.36 & & & $\mathbf{2 4 8}$ & $3-\mathrm{MDBF}$ & & 0.17 \\
\hline
\end{tabular}

Table S50. Ketones Detected in SP $\mathrm{CHC}_{\mathrm{L}}$ and SPCHC-F

\begin{tabular}{cccccc}
\hline peak & ketone & RC in $\mathrm{SP}_{\mathrm{CHC}-\mathrm{F}}$ & peak & ketone & RC in SP $\mathrm{CHC}_{\mathrm{L}}$ \\
\hline $\mathbf{7 8}$ & pulegone & 0.24 & $\mathbf{3 1 2}$ & benzoanthracen-7-one & 0.09 \\
$\mathbf{8 7}$ & indan-2-one & 1.35 & & & \\
\hline
\end{tabular}

Table S51. OOCOCs Detected in SP $\mathrm{NCHC}_{\mathrm{C}}, \mathrm{SP}_{\mathrm{CHC}-\mathrm{L}}$, and SPCHC-F

\begin{tabular}{|c|c|c|c|c|}
\hline \multirow{2}{*}{ peak } & \multirow{2}{*}{ OOCOC } & \multicolumn{3}{|c|}{$\mathrm{RC}$} \\
\hline & & $\mathrm{SP}_{\mathrm{NCHC}}$ & $\mathrm{SP}_{\mathrm{CHC}-\mathrm{L}}$ & $\mathrm{SP}_{\mathrm{CHC}-\mathrm{F}}$ \\
\hline 51 & octahydrobenzofuran & & 0.04 & \\
\hline 66 & 2,3-dihydrobenzofuran & & 0.12 & 0.13 \\
\hline 171 & 1,2,3,4-tetrahydrodibenzofuran & & 0.09 & 0.65 \\
\hline 180 & 2-ethoxynaphthalene & & 0.19 & \\
\hline 313 & naphtho[2,1,8,7-klmn]xanthene & 0.11 & & \\
\hline 187 & 3-phenoxyphenol & & & 1.30 \\
\hline
\end{tabular}

Table S52. PTNSAs Detected in SPNCHC

\begin{tabular}{cccccc}
\hline peak & PTNSA & RC & peak & PTNSA & RC \\
\hline $\mathbf{1}$ & pyridine & 0.01 & $\mathbf{2 8 8}$ & acenaphtho[1,2- $b$ ]pyridine & 0.08 \\
$\mathbf{2 5 2}$ & indeno[1,2-b]pyridine & 0.43 & & & \\
\hline
\end{tabular}

Table S53. ASPs Detected in SPNCHC

\begin{tabular}{cccccc}
\hline peak & ASP & RC & peak & ASP & RC \\
\hline $\mathbf{1 1}$ & 2-methylpyridine & 0.01 & $\mathbf{3 7}$ & 3,4-dimethylpyridine & 0.02 \\
$\mathbf{2 0}$ & 3-methylpyridine & 0.01 & $\mathbf{4 2}$ & 4-ethylpyridine & 0.01 \\
$\mathbf{2 5}$ & 2,6-dimethylpyridine & 0.01 & $\mathbf{5 9}$ & 2,3,6-trimethylpyridine & 0.01 \\
\hline
\end{tabular}

Table S54. QTNSAs Detected in $\mathrm{SP}_{\mathrm{NCHC}}$

\begin{tabular}{ccccccccc}
\hline peak & QTNSA & RC & peak & QTNSA & RC & peak & QTNSA & RC \\
\hline $\mathbf{1 3 3}$ & quinoline & 0.33 & $\mathbf{2 4 6}$ & phenanthridine & 0.06 & $\mathbf{2 8 0}$ & indeno[1,2,3-de $]$ quinoline & 0.06 \\
$\mathbf{1 3 9}$ & isoquinoline & 0.05 & $\mathbf{2 5 0}$ & benzo[f]quinoline & 0.09 & $\mathbf{3 0 6}$ & naphtho[2,3- $h]$ quinoline & 0.09 \\
\hline
\end{tabular}


Table S55. MQs Detected in SP $\mathrm{NCHC}_{\mathrm{C}}$

\begin{tabular}{ccccccccc}
\hline peak & compound & RC & peak & compound & RC & peak & compound & RC \\
\hline $\mathbf{1 5 0}$ & 2-MQ & 0.05 & $\mathbf{1 5 8}$ & 6-MQ & 0.01 & $\mathbf{1 6 7}$ & 5-MQ & 0.01 \\
$\mathbf{1 5 5}$ & 7-MQ & 0.02 & $\mathbf{1 6 4}$ & 8-MQ & 0.02 & & & \\
\hline
\end{tabular}

Table S56. ANs ${ }^{\mathrm{II}}$ Detected in $\mathrm{SP}_{\mathrm{NCHC}}$

\begin{tabular}{cccccc}
\hline peak & AN $^{\mathrm{II}}$ & $\mathrm{RC}$ & peak & $\mathrm{AN}^{\mathrm{II}}$ & $\mathrm{RC}$ \\
\hline $\mathbf{5 2}$ & benzonitrile & 0.05 & $\mathbf{2 0 0}$ & 2-naphthonitrile & 0.09 \\
\hline
\end{tabular}

Table S57. Indoles Detected in SPNCHC

\begin{tabular}{ccccccccc}
\hline peak & compound & RC & peak & compound & RC & peak & compound & RC \\
\hline $\mathbf{1 4 7}$ & indole & 0.15 & $\mathbf{1 6 6}$ & 4-methylindole & 0.01 & $\mathbf{1 7 2}$ & 6-methylindole & 0.01 \\
\hline
\end{tabular}

Table S58. MCs ${ }^{\text {II }}$ Detected in SPNCHC

\begin{tabular}{cccccc}
\hline peak & MC $^{\mathrm{II}}$ & $\mathrm{RC}$ & peak & $\mathrm{MC}^{\mathrm{II}}$ & $\mathrm{RC}$ \\
\hline $\mathbf{2 6 5}$ & 2-methylcarbazole & 0.04 & $\mathbf{2 6 6}$ & 9-methylcarbazole & 0.04 \\
\hline
\end{tabular}

Table S59. BCs Detected in SPNCHC

\begin{tabular}{cccccc}
\hline peak & BC & RC & peak & BC & RC \\
\hline $\mathbf{2 9 1}$ & benzo $[d]$ carbazole & 0.04 & $\mathbf{3 1 4}$ & benzo $[b]$ carbazole & 0.11 \\
\hline \multicolumn{7}{c}{ Table S60. } \\
\hline \multicolumn{7}{c}{ DMPs ${ }^{\text {II }}$} & Detected in SPNCHC \\
\hline peak & compound & RC & peak & compound & RC \\
\hline $\mathbf{2 8 1}$ & $2,9-D M P^{\text {II }}$ & 0.20 & $\mathbf{2 8 4}$ & $4,7-D M P ~$ & 0.14 \\
\hline
\end{tabular}

Table S61. ONCOCs Detected in SPNCHC, SPCHC-L, and SPCHC-F

\begin{tabular}{ccccc}
\hline \multirow{2}{*}{ peak } & ONCOC & \multicolumn{3}{c}{$\mathrm{RC}$} \\
\cline { 3 - 5 } & & $\mathrm{SP}_{\mathrm{NCHC}}$ & $\mathrm{SP}_{\mathrm{CHC}-\mathrm{L}}$ & $\mathrm{SP}_{\mathrm{CHC}-\mathrm{F}}$ \\
\hline $\mathbf{4 8}$ & aniline & 0.01 & & \\
$\mathbf{9 3}$ & $(4 \mathrm{a} R, 8 \mathrm{a} R)$-1-methyldecahydroquinoline & & 0.08 & \\
$\mathbf{1 1 7}$ & 2,3 -diethylpyrazine & & 0.39 & \\
$\mathbf{1 5 9}$ & 2-ethyl-1H-pyrrolo[2,3-b]pyridine & & & 0.79 \\
$\mathbf{2 0 5}$ & $9 H$-pyrido[3,4- $b$ ]indole & 2.31 & & \\
\hline
\end{tabular}

Table S62. TTNSA Detected in SPNCHC

\begin{tabular}{cccccc}
\hline peak & TTNSA & RC & peak & TTNSA & RC \\
\hline $\mathbf{1 1 9}$ & benzothiophene & 0.73 & $\mathbf{3 0 4}$ & benzonaphtho[2,1-d]thiophene & 0.22 \\
$\mathbf{2 4 2}$ & dibenzothiophene & 0.64 & $\mathbf{3 0 7}$ & benzonaphtho[2,3-d]thiophene & 0.05 \\
$\mathbf{2 4 7}$ & naphtho[2,3-b]thiophene & 0.05 & $\mathbf{3 0 8}$ & benzonaphtho[1,2-d]thiophene & 0.03 \\
\hline
\end{tabular}

Table S63. TTMA Detected in SPNCHC

\begin{tabular}{cccccc}
\hline peak & TTMA & RC & peak & TTMA & RC \\
\hline $\mathbf{4}$ & 2-methylthiophene & 0.01 & $\mathbf{2 5 8}$ & 4-methyldibenzothiophene & 0.08 \\
$\mathbf{1 4 9}$ & 3-methylbenzothiophene & 0.06 & & & \\
\hline
\end{tabular}




\section{Table S64}

Classification of the group components detected in the SPs with GC/MS.

\begin{tabular}{|c|c|c|c|c|c|}
\hline \multicolumn{2}{|l|}{ group component } & \multirow{2}{*}{ from Table } & \multicolumn{2}{|l|}{ group component } & \multirow{2}{*}{ from Table } \\
\hline full name & nomenclature & & full name & nomenclature & \\
\hline normal alkanes & NAs & Table S1 & dimethylnaphthalenes & DMNs & Table S26 \\
\hline alkylcyclohexanes & $\mathrm{ACHs}^{\mathrm{I}}$ & Table S2 & trimethylnaphthalenes & TMNs & Table S27 \\
\hline dimethylcyclohexanes & DMCHs & Table S3 & methylanthracenes & MAs & Table S28 \\
\hline 1-ethyl(methyl)cyclohexanes & EMCHs & Table S4 & dimethylanthracenes & DMAs & Table S29 \\
\hline 1-methyl(propyl)cyclohexane & MPCHs & Table S5 & methylphenanthrenes & MPs & Table S30 \\
\hline 1-isopropyl(methyl)cyclohexane & IPMCHs & Table S6 & dimethylphenanthrenes & DMPs $^{\mathrm{I}}$ & Table S31 \\
\hline trimethylcyclohexanes & TMCHs & Table S7 & methylfluoranthenes & $\mathrm{MFs}^{\mathrm{II}}$ & Table S32 \\
\hline non-substituted polycyclanes & NSPCs & Table S8 & methyl-substituted pyrenes & MSPs ${ }^{I}$ & Table S33 \\
\hline alkyldecalins & ADs & Table S9 & methylchrysenes & $\mathrm{MCs}^{\mathrm{I}}$ & Table S34 \\
\hline dimethyldecalins & DMDs & Table S10 & non-substituted hydroarenes & NSHAs & Table S35 \\
\hline methylperhydrophenanthrenes & MPHPs & Table S11 & methylindanes & MIs ${ }^{\mathrm{II}}$ & Table S36 \\
\hline other cyclanes & OCs & Table S12 & dimethylindanes & DMIs & Table S37 \\
\hline alkyl-substituted cyclohex-1-ene & ASCHs & Table S13 & alkyltetralins & ATs & Table S38 \\
\hline non-substituted condensed arenes & NSCAs & Table S14 & dimethyltetralins & DMTs & Table S39 \\
\hline polyaryls & PAs & Table S15 & alkyl-1,2,3,4,5,6,7,8-octahydroarenes & AOHAs & Table S40 \\
\hline single methylene-containing condensed arenes & SMCCAs & Table S16 & other hydroarenes & OHAs & Table S41 \\
\hline alkylbenzenes & $\mathrm{ABs}$ & Table S17 & other arenes & OAs & Table S42 \\
\hline dialkylbenzenes & DABs & Table S18 & cyclohexanols & $\mathrm{CHs}$ & Table S43 \\
\hline trialkylbenzenes & TABs & Table S19 & alkoxycyclohexanes & $\mathrm{ACHs}^{\mathrm{II}}$ & Table S44 \\
\hline tetramethylbenzenes & TMBs & Table S20 & non-substituted arenols & NSAs & Table S45 \\
\hline alkenyl arenes & AAs & Table S21 & methyl-substituted phenols & MSPs ${ }^{I I}$ & Table S46 \\
\hline methylindenes & MIs ${ }^{\mathrm{I}}$ & Table S22 & arenobenzofurans & ABFs & Table S47 \\
\hline methyl-substituted biphenyls & MSBPs & Table S23 & benzofurans & $\mathrm{BFs}$ & Table S48 \\
\hline methylfluorenes & $M F s^{I}$ & Table S24 & methyldibenzofurans & MDBFs & Table S49 \\
\hline alkylnaphthalenes & $\mathrm{ANs}^{\mathrm{I}}$ & Table S25 & ketones & & Table S50 \\
\hline
\end{tabular}




\section{Table S64}

Classification of the group components detected in SPs with GC/MS (continued).

\begin{tabular}{|c|c|c|c|c|c|}
\hline \multicolumn{2}{|l|}{ group component } & \multirow{2}{*}{ from Table } & \multicolumn{2}{|l|}{ group component } & \multirow{2}{*}{ from Table } \\
\hline full name & nomenclature & & full name & nomenclature & \\
\hline other oxygen-containing organic compounds & OOCOCs & Table S51 & methylcarbazoles & MCs ${ }^{I I}$ & Table S58 \\
\hline pyridine-type non-substituted aromatics & PTNSAs & Table S52 & benzocabazoles & $\mathrm{BCs}$ & Table S59 \\
\hline alkyl-substituted pyridines & ASPs & Table S53 & dimethylphenanthrolines & DMPsII & Table S60 \\
\hline quinoline-type non-substituted aromatics & QTNSAs & Table S54 & other nitrogen-containing organic compounds & ONCOCs & Table S61 \\
\hline methylquinolines & MQs & Table S55 & thiophene-type non-substituted aromatics & TTNSAs & Table S62 \\
\hline arenonitrile & $\mathrm{ANs}^{\mathrm{II}}$ & Table S56 & thiophene-type methylaromatics & TTMAs & Table S63 \\
\hline indoles & & Table S57 & & & \\
\hline
\end{tabular}




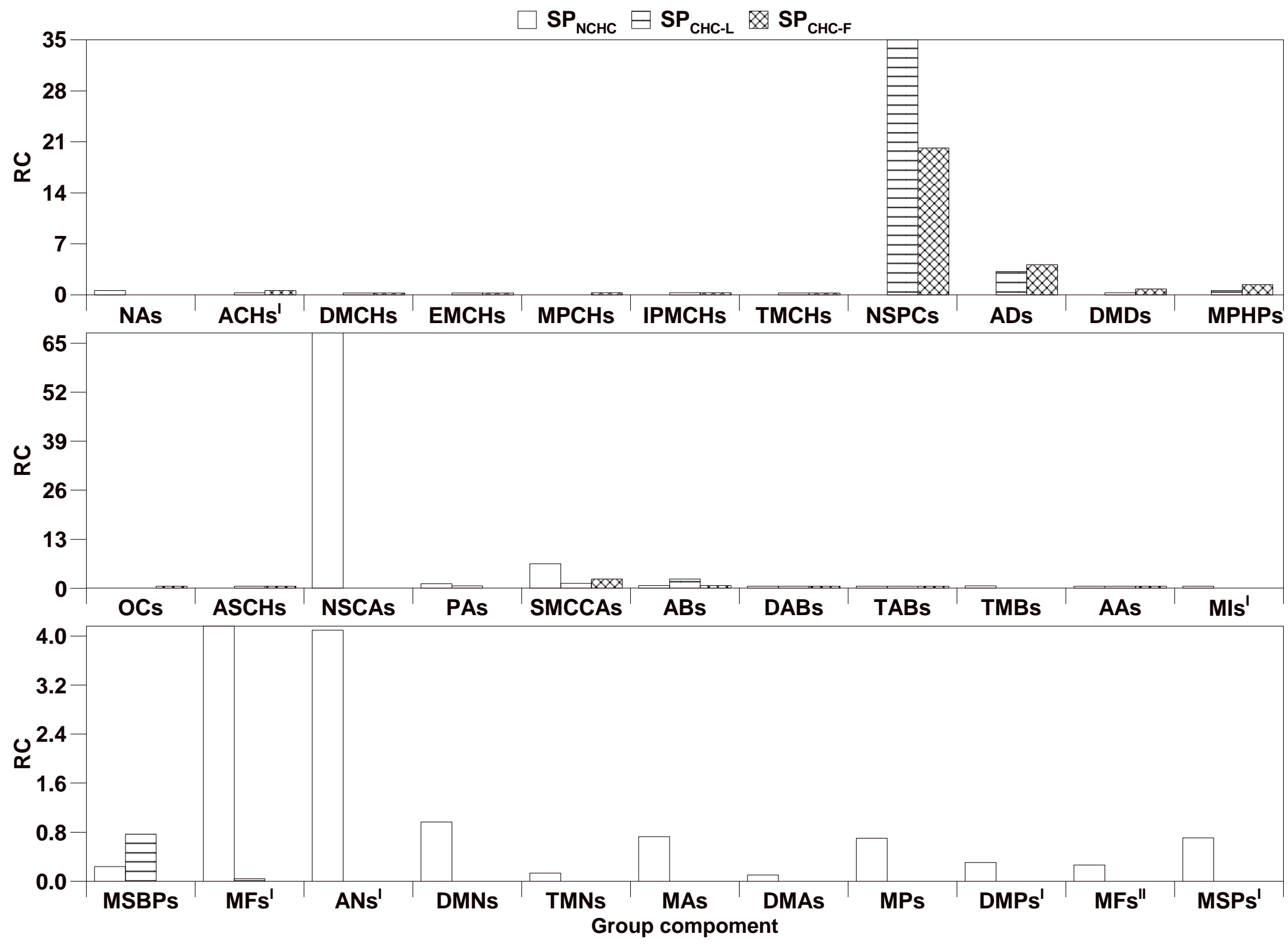

Figure S9. Distribution of group compounds in SPs. 


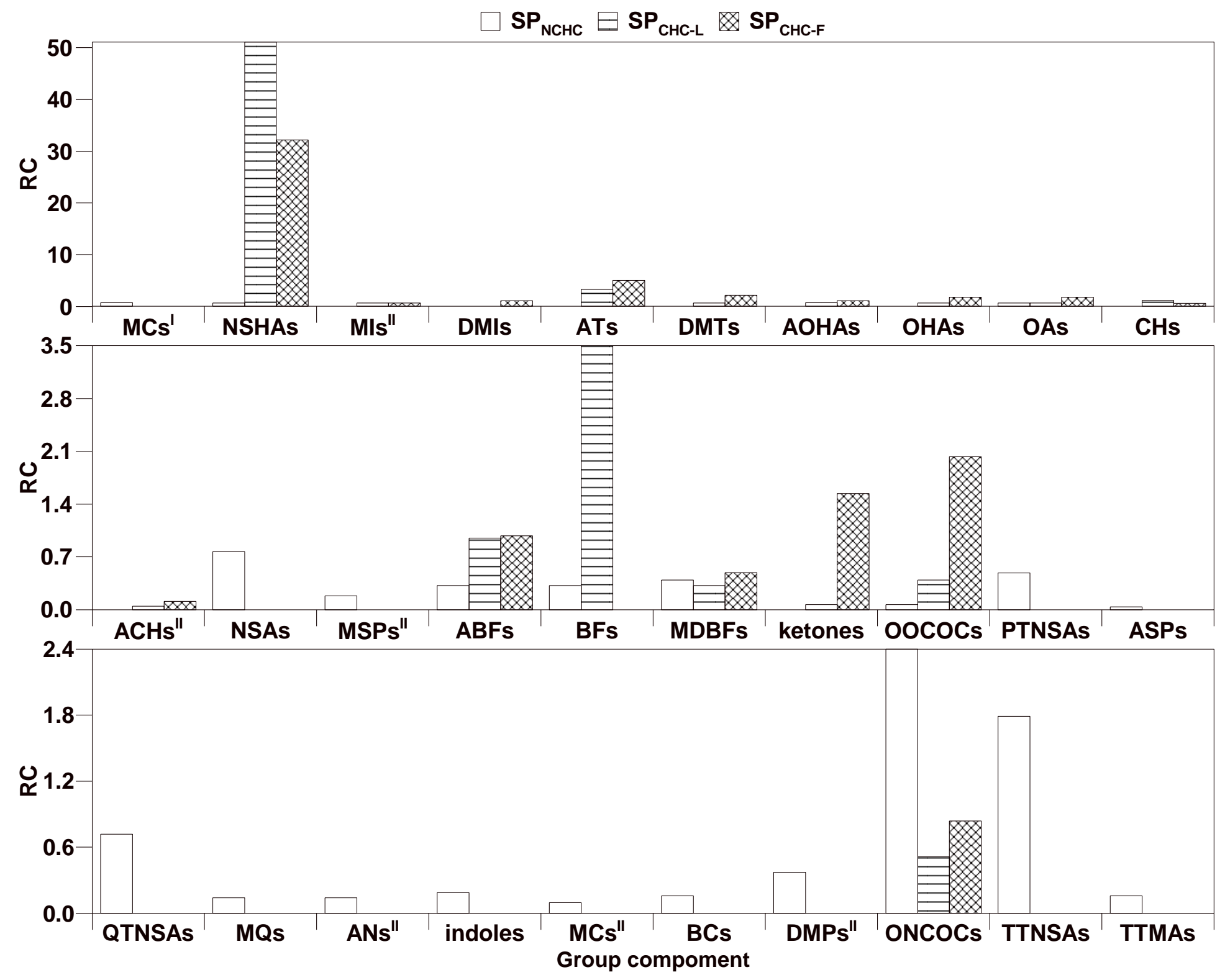

Figure S9. Distribution of group compounds in SPs (continued). 

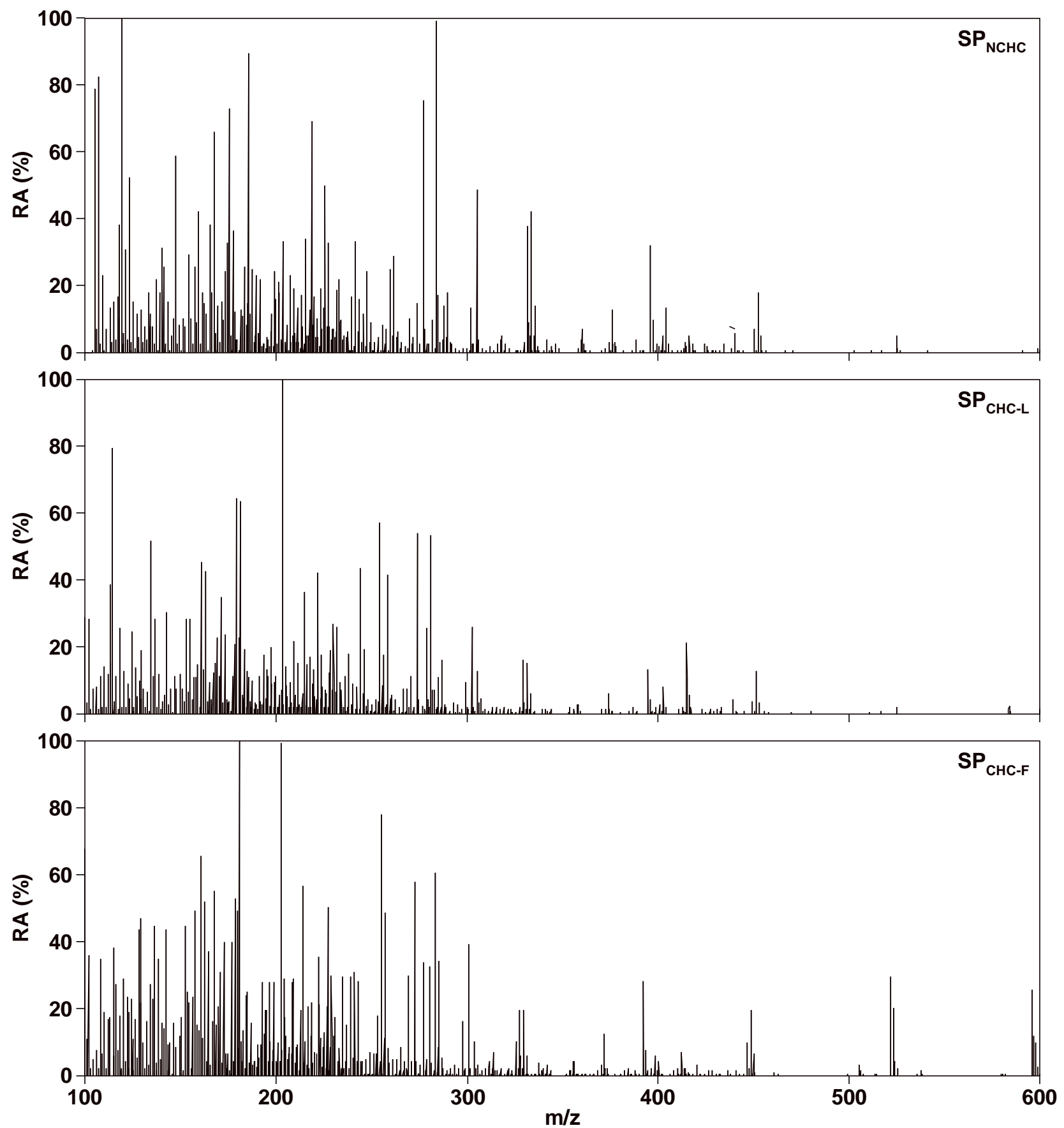

Figure S10. Broadband mass spectra from the analysis of the SPs with QEOTMS. 